\title{
Global Gravitational Anomalies
}

\author{
Edward Witten* \\ Joseph Henry Laboratories, Princeton University, Princeton, NJ 08544, USA
}

\begin{abstract}
A general formula for global gauge and gravitational anomalies is derived. It is used to show that the anomaly free supergravity and superstring theories in ten dimensions are all free of global anomalies that might have ruined their consistency. However, it is shown that global anomalies lead to some restrictions on allowed compactifications of these theories. For example, in the case of $\mathrm{O}(32)$ superstring theory, it is shown that a global anomaly related to $\pi_{7}(\mathrm{O}(32))$ leads to a Dirac-like quantization condition for the field strength of the antisymmetric tensor field.

Related to global anomalies is the question of the number of fermion zero modes in an instanton field. It is argued that the relevant gravitational instantons are exotic spheres. It is shown that the number of fermion zero modes in an instanton field is always even in ten dimensional supergravity.
\end{abstract}

\section{Introduction}

Anoamlies which arise in perturbation theory [1] can ruin the consistency of quantum field theories. Cancellation of such anomalies is a significant restriction on models in four dimensions [2], and even more so in higher dimensions [3], especially when gravitational and mixed gauge-gravitational anomalies are included [4]. Recently Green and Schwarz have exhibited a new mechanism for anomaly cancellation in higher dimensions [5], which leads to the existence of $n=1$ supersymmetric theories with "miraculous" anomaly cancellation in ten dimensions (and $\mathrm{O}(32)$ or $E_{8} \times E_{8}$ gauge group). These theories are particularly attractive because they are the low energy limits of consistent superstring theories [6]. (The original discovery involved $\mathrm{O}(32)$, but a superstring theory based on $E_{8}$ $\times E_{8}$ has been discovered [7].) Such "miraculous" cancellation of anomalies also occurs [4] in the $n=2$ chiral supersymmetry theory in ten dimensions, but that

* Supported in part by NSF Grant No. PHY80-19754 
theory, while also the low energy limit of a superstring theory, seems less attractive phenomenologically.

When perturbative anomalies cancel, this means that the effective action is invariant under gauge and coordinate transformations that can be reached continuously from the identity. One must still ask whether the effective action is invariant under gauge or coordinate transformations that are not continuously connected to the identity. Lack of such invariance leads, for instance, to quantization of a certain mass parameter in $2+1$ dimensions [8]. It also leads to the inconsistency of an SU(2) gauge theory with an odd number of fermion doublets in $3+1$ dimensions [9]. In this paper, gauge or coordinate transformations that cannot be reached continuously from the identity will be called global gauge or coordinate transformation, and lack of invariance of the effective action under such transformations will be referred to as a global anomaly.

Global anomalies can enter in general relativity; for instance, in $8 k$ or $8 k+1$ dimensions, a theory with an odd number of spin 1/2 Majorana fermions coupled to gravity has a global anomaly [4]. In parity conserving theories, the Euclidean effective action is always real. Global anomalies can then involve only the sign of the effective action, and can be investigated by counting the number of fermion zero modes modulo two. In parity-violating theories, the analysis of global anomalies is more subtle since the effective action is complex, and one must keep track of its phase, not just its sign. Of course, in parity-violating theories, perturbative anomalies may occur. It really only makes sense to discuss global anomalies if the perturbative anomalies cancel (since a global coordinate or gauge transformation, defined by its topological class, is only well-defined modulo a local transformation). Evaluation of global anomalies in parity-violating theories in which local anomalies cancel is an interesting and subtle problem, raised in [4]. In certain cases $[10,11]$, global gauge anomalies can be calculated by embedding them in the local anomaly of a larger group. This method also gives interesting results for gauge theories in which anomalies are cancelled by the Green-Schwarz mechanism [12]. However, it does not appear possible to evaluate global gravitational anomalies in this way. Evaluation of global gravitational anomalies in the ten dimensional supergravity theories is important, because in ten dimensional space-time the group of (orientation-preserving) diffeomorphisms (with compact support) is disconnected, having 992 components [13, 19]. One must ask whether lack of invariance under some of the 991 global coordinate transformations spoils the consistency of some of the ten dimensional theories that are free of perturbative anomalies. The main result of this paper is to show that this does not occur for the $\mathrm{O}(32)$ and $E_{8} \times E_{8}$ theories and apparently also does not occur for the chiral $n=2$ theory.

We will not discuss global gauge anomalies for the ten dimensional supergravity theories; the reason for this is that $\pi_{10}(\mathrm{O}(32)) \cong \pi_{10}\left(E_{8} \times E_{8}\right) \cong 0$, so global gauge anomalies cannot arise.

Showing that a given superstring theory reduces at low energies to a field theory free of global or local anomalies does not guarantee that the superstring theory is fully consistent since it is always possible that the string theory has "inherently stringy" anomalies that vanish in the limit of lowly Riemannian geometry. However, anomalies present in the field theory would have to be present 
in the string theory (assuming one studies the right field theory with all relevant terms!), in view of the usual arguments showing that anomalies can be extracted from the long distance behavior of a theory.

Section II of this paper is devoted to mathematical preliminaries. Section III (not strictly necessary for the later development) gives a simplified and partial account of global anomalies in terms of instantons. Section IV gives a general analysis of global anomalies for theories in which the anomalies cancel out of the sum of one loop diagrams. Application to the chiral $n=2$ supergravity theory indicate it has no global anomalies in ten dimensions, but the conclusion is not quite rigorous because of physical and mathematical uncertainties about antisymmetric tensor fields. Section $\mathrm{V}$ gives a very general global anomaly formula for the $\mathrm{O}(32)$ and $E_{8} \times E_{8}$ theories. It is shown that these theories have no global anomalies in ten dimensions, before compactification. However, global anomalies may put restrictions on compactifications of these theories. A first example of this kind is developed in Sect. VI; it is a Dirac-like quantization condition for the field strength of the antisymmetric tensor that holds in the O(32) case. Section VII contains concluding comments.

Although we will not explore this subject here, it is worth mentioning that global gravitational anomalies enter in string theory in an entirely different way. At tree level, one encounters in the theory of closed strings a path integral on a world sheet that is topologically a sphere. The orientation preserving diffeomorphism group of the sphere is connected, and invariance under this group is required in quantizing the free string. At one loop level, the world sheet is a torus, whose diffeomorphism group is far from connected. The group of components of the orientation-preserving diffeomorphism group of the torus is called the modular group. Failure of modular invariance, which eliminates some would-be gauge groups in the models of [7], can be construed as a global gravitational anomaly on the string world sheet. This might have non-trivial generalizations in higher orders.

\section{Mathematical Background}

Consider a theory in $n$ space-time dimensions, for which we consider a Euclidean formulation on a sphere $S^{n}$. The group of parity-conserving diffeomorphisms of $S^{n}$ may contain an element $\pi$ not continuously connected to the identity. We wish to ask whether the effective action of our theory is invariant under $\pi .^{1}$

A diffeomorphism $\pi$ of the $n$-sphere can be studied by relating it to a manifold of $n+1$ dimensions which is closely related to the standard $n+1$ sphere $S^{n+1}$. This manifold, which we will call $S_{n}^{n+1}$, is defined as follows [14] (Fig. 1). Take the standard $n+1$ sphere and cut it in half at the equator; then glue the two halves back together after transforming the boundary of the northern hemisphere by $\pi$. [In other words, a point $x$ on the boundary of the southern hemisphere is glued

1 It is highly plausible that lack of such invariance would signify the inconsistency of the theory under study, but properly this should be deduced from canonical considerations. This has been done in the gauge theory case [9] and a similar discussion is possible in the gravitational case, as we will briefly discuss in Sect. III 
(a)

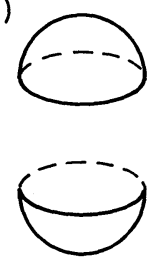

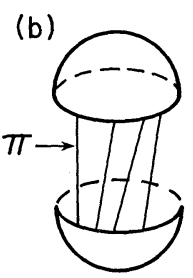

Fig. 1a and b. To make a potentially exotic sphere, an ordinary sphere is cut in two on the equator. The two hemispheres are then glued back together along the equator after making a diffeomorphism $\pi$ of the boundary of the northern hemisphere

onto a point $\pi(x)$ on the boundary of the northern hemisphere'] It is obvious that if $\pi$ is continuously connected to the identity, then $S_{\pi}^{n+1}$ and $S^{n+1}$ are topologically equivalent. However, if $\pi$ is not continuously connected to the identity, then $S_{\pi}^{n+1}$ and $S^{n+1}$ turn out to differ in a subtle way.

Two manifolds $A$ and $B$ are said to be homeomorphic if there is a continuous, one to one mapping from $A$ to $B$. If there is a differentiable, one to one mapping from $A$ to $B$, then $A$ and $B$ are said to be diffeomorphic. In the case of spheres, a manifold which is homeomorphic but not diffeomorphic to the ordinary sphere $S^{n+1}$ is said to be an "exotic sphere." It is relatively easy to prove [14] that $S_{\pi}^{n+1}$ is always homeomorphic to $S^{n+1}$ (and diffeomorphic to it if $\pi$ is topological trivial). With more difficulty it can be proved that $S_{\pi}^{n+1}$ is diffeomorphic to $S^{n+1}$ only if $\pi$ is topologically trivial [15]. Thus $S_{\pi}^{n+1}$ is an exotic sphere if and only if $\pi$ is not continuously connected to the identity. It can also be proved [13], with some difficulty, that every exotic $n+1$ sphere is $S_{\pi}^{n+1}$ for some $\pi$. Thus, exotic $n+1$ spheres and topological classes of diffeomorphisms of the $n$ sphere are in one to one correspondence.

It is almost always easier to construct (and study) an exotic $n+1$ sphere $\widetilde{S}^{n+1}$ than to construct directly the associated diffeomorphism of $S^{n}$. Moreover, the easiest way to study an exotic $n+1$ sphere $\widetilde{S}^{n+1}$ is usually to study an oriented $n+2$ manifold $B$ which has $\tilde{S}^{n+1}$ for boundary. ( $B$ will always exist since the Pontryagin and Stiefel-Whitney numbers of an exotic sphere are zero.)

Before giving examples of how this is done, let us first discuss some facts about oriented manifolds with boundary. To be specific (since it is the main case that will interest us later) consider an oriented 12 manifold $B$ with boundary $\partial B$. Let $B$ be endowed with a Riemannian metric $g$, and let $R$ be the associated curvature two form. If $B$ is a closed manifold, with no boundary, so $\partial B=0$, then the following integrals are topological invariants:

$$
\begin{gathered}
I_{1}=\int_{B}\left(\operatorname{Tr} R^{2}\right)^{3}, \\
I_{2}=\int_{B}\left(\operatorname{Tr} R^{2}\right) \cdot\left(\operatorname{Tr} R^{4}\right), \\
I_{3}=\int_{B} \operatorname{Tr} R^{6} .
\end{gathered}
$$


To show that these are topological invariants, one notes for instance that under a change in the metric of $B$, the change in $\operatorname{Tr} R^{2}$ is $\delta \operatorname{Tr} R^{2}=d \Lambda$ with some globally defined $\Lambda$; hence

$$
\delta I_{1}=3 \int_{B} d \Lambda \cdot\left(\operatorname{Tr} R^{2}\right)^{2}=-3 \int_{B} \Lambda d\left(\left(\operatorname{Tr} R^{2}\right)^{2}\right)=0 ;
$$

here we have integrated by parts, and used the fact that $\partial B=0$ and that $d \operatorname{Tr} R^{2}=0$.

Now we wish to generalize $I_{1}, I_{2}$, and $I_{3}$ - insofar as possible - to give topological invariants of a manifold $B$ with non-empty boundary $\partial B$. This is possible only under certain conditions.

In general, the equation $\operatorname{Tr} R^{2}=d H$ cannot be solved globally on $B$; if it can be solved, $I_{1}$ and $I_{2}$ vanish. However, it may be possible to solve $\operatorname{Tr} R^{2}=d H$ on $\partial B$. If so, we generalize $I_{1}$ and $I_{2}$ to

$$
\begin{aligned}
& \bar{I}_{1}=\int_{B}\left(\operatorname{Tr} R^{2}\right)^{3}-\int_{\partial B} H\left(\operatorname{Tr} R^{2}\right)^{2}, \\
& \bar{I}_{2}=\int_{B} \operatorname{Tr} R^{2} \operatorname{Tr} R^{4}-\int_{\partial B} H \operatorname{Tr} R^{4} .
\end{aligned}
$$

To see that these are invariant under a change in metric of $B$, note that if $\delta \operatorname{Tr} R^{2}$ $=d \Lambda$, then the choice $\delta H=\Lambda$ preserves the requirement that $\operatorname{Tr} R^{2}=d H$ on $\partial B$, and clearly then $\delta \bar{I}_{1}=\delta \bar{I}_{2}=0$. This does not quite show that $\bar{I}_{1}$ and $\bar{I}_{2}$ are welldefined, because we must show that $\bar{I}_{1}$ and $\bar{I}_{2}$ are independent of the choice of $H$, as long as $d H=\operatorname{Tr} R^{2}$.

If $d H=d H^{\prime}=\operatorname{Tr} R^{2}$, then $d\left(H-H^{\prime}\right)=0$. In changing from $H$ to $H^{\prime}$, the change in $\bar{I}_{1}$ is

$$
\Delta \bar{I}_{1}=\int_{\partial B}\left(H-H^{\prime}\right)\left(\operatorname{Tr} R^{2}\right)^{2}=\int_{\partial B}\left(H-H^{\prime}\right) \cdot d H \cdot \operatorname{Tr} R^{2}=0,
$$

where in the last step we integrate by parts and use $d\left(H-H^{\prime}\right)=d \operatorname{Tr} R^{2}=0$. Thus $\bar{I}_{1}$ is well-defined. As for $\bar{I}_{2}$, it changes by

$$
\Delta \bar{I}_{2}=\int_{\partial B}\left(H-H^{\prime}\right) \operatorname{Tr} R^{4} .
$$

In general, this does not vanish. But if $\operatorname{Tr} R^{4}=d K$ on $\partial B$, then

$$
\Delta \overline{I_{2}}=\int_{\partial B}\left(H-H^{\prime}\right) d K=-\int_{\partial B} d\left(H-H^{\prime}\right) \cdot K=0,
$$

In conclusion, then, $I_{1}$ can be generalized to a topological invariant of a manifold $B$ with boundary if $\operatorname{Tr} R^{2}=d H$ on $\partial B$. $I_{2}$ can be so generalized if $\operatorname{Tr} R^{2}$ $=d H$ and $\operatorname{Tr} R^{4}=d K$ on $\partial B$. However, there is not analogue of $I_{3}$ for manifolds with boundary. This observation meshes in an interesting way with the GreenSchwarz anomaly cancellation mechanism, as we will see in Sect. V.

In Sect. VI, we will encounter a situation in which $\operatorname{Tr} R^{4} \neq d K$ on $\partial B$, so that $\bar{I}_{2}$ depends on the choice of $H$; however, in the physical problem, there will be a natural and physically motivated choice of $H$. Under such conditions, $\bar{I}_{2}$ is meaningful as a functional of $H$ and the metric of $\partial B$ (independent of the choice of metric of $B$ away from $\partial B$ ).

It is useful to note that $\bar{I}_{1}$ and $\bar{I}_{2}$ are not merely independent of the metric of $B$. They are also independent of the connection on the tangent bundle of $B$, even if one 
considers connections with torsion. A manifold is said to be parallelizable if it admits a connection (perhaps with torsion) for which $R=0$ everywhere; obviously, in that case $\bar{I}_{1}=\bar{I}_{2}=0$.

We are now nearly ready to discuss exotic spheres. We will follow Milnor's presentation [16] of a refinement by Eells and Kuiper [17] of Milnor's original treatment. We will consider both the seven and eleven dimensional cases, though our main interest is eleven dimensions.

For a closed eight dimensional manifold $B$, define

$$
\begin{gathered}
p_{1}^{2}=\frac{1}{(2 \pi)^{4}} \int_{B} \frac{1}{4}\left(\operatorname{Tr} R^{2}\right)^{2}, \\
p_{2}=\frac{1}{(2 \pi)^{4}} \int_{B}\left(\frac{1}{8}\left(\operatorname{Tr} R^{2}\right)^{2}-\frac{1}{4} \operatorname{Tr} R^{4}\right) .
\end{gathered}
$$

In view of our previous discussion, $p_{2}$ has no generalization for manifolds $B$ with boundary, but $p_{1}^{2}$ does as long as $\operatorname{Tr} R^{2}=d H$ on the boundary of $B$; we will denote this generalization as $p_{1}^{2}$ whether or not $B$ has a boundary.

For an eight dimensional spin manifold, the index of the Dirac operator is

$$
\text { index }(i \not D)=\frac{1}{5760}\left(7 p_{1}^{2}-4 p_{2}\right),
$$

while the signature $\sigma$ is

$$
\sigma=-\frac{1}{45} p_{1}^{2}+\frac{7}{45} p_{2}
$$

These combine into

$$
\text { index }(i \not D)=\frac{1}{896}\left(p_{1}^{2}-4 \sigma\right) \text {. }
$$

Since the Dirac index is an integer, it follows that $\frac{1}{896}\left(p_{1}^{2}-4 \sigma\right)$ is an integer for any closed eight manifold.

Now consider a seven dimensional spin manifold $M$ with $\operatorname{Tr} R^{2}=d H$ on $M$. If $M$ is the boundary of some oriented eight dimensional spin manifold $B$, let

$$
\lambda(M)=\frac{1}{896}\left(p_{1}^{2}(B)-4 \sigma(B)\right) \bmod 1 .
$$

The claim is that modulo $1, \lambda$ depends on $M$ only and not $B$, though the right-hand side of (9) appears to depend on $B$ as well as $M$. To prove this, let $B$ and $B^{\prime}$ be two different oriented spin manifolds that bound $M$. Then by gluing $B$ and $B^{\prime}$ together along $M$ (with opposite orientation so that the boundary term in $\lambda$ will cancel) we get a closed eight manifold $X$ which may be denoted $X=B+\left(-B^{\prime}\right)$. If $\lambda=\frac{1}{896}\left(p_{1}^{2}(B)-4 \sigma(B)\right), \lambda^{\prime}=\frac{1}{896}\left(p_{1}^{2}\left(B^{\prime}\right)-4 \sigma\left(B^{\prime}\right)\right)$, then $\lambda-\lambda^{\prime}=\frac{1}{896}\left(p_{1}^{2}(X)-4 \sigma(X)\right)$, which by our previous comments must be an integer. Hence $\lambda=\lambda^{\prime} \bmod 1$.

Now, in $4 k-1$ dimensions for any $k \geqq 2$, Milnor has given [18] an elementary geometrical construction of a manifold $Q$ which is homeomorphic to the standard sphere and bounds a parallelizable manifold $B$ of signature 8 . (The construction makes use of an $8 \times 8$ matrix which happens to be the Cartan matrix of the group $E_{8}$.) Now, consider the case of seven dimensions, $k=2$. Since $B$ is parallelizable, $p_{1}^{2}(B)=0$, so $\lambda(Q)=-4 \sigma(B) / 896=-1 / 28$. Since $\lambda=0$ for the standard sphere, $Q$ must be an exotic sphere. By taking $n$ connected copies of $Q$ for $1 \leqq n \leqq 27$, one makes 27 exotic spheres which must be non-diffeomorphic to one another since 
they have different $\lambda$. With more work one can show that these are all of the exotic seven spheres.

In eleven dimensions, the facts are similar. For closed twelve manifolds, define

$$
\begin{gathered}
p_{1}^{3}=\frac{1}{(2 \pi)^{6}} \int\left[\frac{1}{8}\left(\operatorname{Tr} R^{2}\right)^{3}\right] \\
p_{1} p_{2}=\frac{1}{(2 \pi)^{6}} \int\left[\frac{1}{8}\left(\operatorname{Tr} R^{2}\right)\left(\operatorname{Tr} R^{4}\right)-\frac{1}{16}\left(\operatorname{Tr} R^{2}\right)^{3}\right] \\
p_{3}=\frac{1}{(2 \pi)^{6}} \int\left[-\frac{1}{6} \operatorname{Tr} R^{6}+\frac{1}{8} \operatorname{Tr} R^{2} \operatorname{Tr} R^{4}-\frac{1}{48}\left(\operatorname{Tr} R^{2}\right)^{3}\right]
\end{gathered}
$$

We have

$$
\begin{aligned}
\operatorname{index}(i \not D) & =\frac{1}{2^{6}}\left(-\frac{31}{15120} p_{1}^{3}+\frac{11}{3780} p_{1} p_{2}-\frac{1}{945} p_{3}\right) \\
\sigma & =\left(\frac{2}{945} p_{1}^{3}-\frac{13}{945} p_{1} p_{2}+\frac{62}{945} p_{3}\right) .
\end{aligned}
$$

This can be rearranged to give

$$
\frac{1}{2} \operatorname{index}(i \not D)=\left(\alpha p_{1}^{3}+\beta p_{1} p_{2}-\frac{\sigma}{8 \cdot 992}\right),
$$

where $\alpha$ and $\beta$ are certain rational numbers. As we will see later, half the Dirac index is always an integer in 12 dimensions. Hence $\left(a p_{1}^{3}+\beta p_{1} p_{2}-\frac{\sigma}{8 \cdot 992}\right)$ is an integer for closed twelve dimensional spin manifolds.

Let $M$ be an eleven dimensional spin manifold with $\operatorname{Tr} R^{2}=d H, \operatorname{Tr} R^{4}=d K$. If $M$ is the boundary of a spin manifold $B$, let

$$
\lambda(M)=\left(\alpha p_{1}^{3}(B)+\beta p_{1} p_{2}(B)-\frac{\sigma(B)}{8 \cdot 992}\right) .
$$

It follows as before that modulo $1, \lambda$ depends only on $M$ and not on the choice of $B$.

In this case, Milnor's manifold $Q$ which is homeomorphic to the standard twelve sphere and bounds a parallelizable manifold of $\sigma=8$ has $\lambda=\frac{-1}{992}$, so it is again an exotic sphere. By taking connected sums of $Q$ one gets a family of 991 exotic eleven spheres, and it can be shown that there are not others [19].

We will see in Sects. IV and V that the facts just cited are adequate for calculating global gravitational anomalies (on the standard spheres) in six or ten dimensions. To calculate global gravitational anomalies in fourteen dimensions would be more complicated, however (should this be required in the future), since there are exotic fifteen spheres that do not bound parallelizable manifolds [19].

Several further mathematical comments are in order. So far we have studied a diffeomorphism $\pi$ of the $n$ sphere in terms of a related "sphere" $S_{\pi}^{n+1}$. There is an alternative and closely related construction that has two virtues: it is more general, and it is closer to our needs in later sections.

Let $M$ be any oriented $n$ manifold, and $\pi$ an orientation preserving diffeomorphism of $M$ (or $\pi$ may be a gauge transformation on some vector bundle 


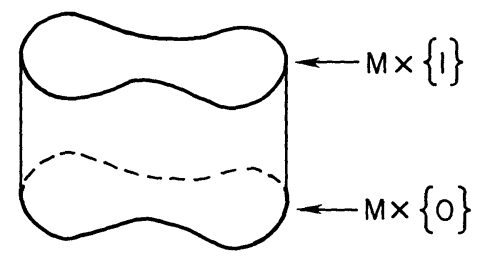

Fig. 2. A cylinder $M \times I, M$ being some manifold and $I$ the unit interval. The cylinder can be made into a closed manifold $\left(M \times S^{1}\right)_{\pi}$ by identifying $(x, 0)$ with $(\pi(x), 1)$ for any $x \in M$. If $\pi$ is the identity, $\left(M \times S^{1}\right)_{\pi}$ is the usual product $M \times S^{1}$
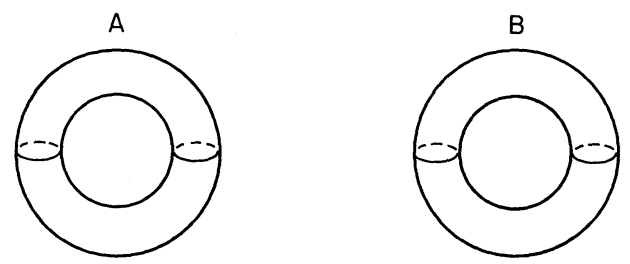

(a)

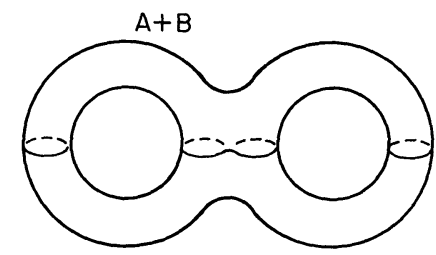

(b)

Fig. 3a and b. The connected sum of two manifolds $\mathbf{A}$ and $\mathbf{B}$ is made by cutting a ball out of $\mathbf{A}$ and $\mathbf{B}$ and gluing them together. If $\mathbf{A}$ and $\mathbf{B}$ are tori [Part (a)] then $\mathbf{A}+\mathbf{B}$ is a surface with two handles [Part (b)]

over $M$, if we wish to investigate global gauge anomalies; or $\pi$ may be a combined gauge and coordinate transformation to study combined global anomalies). Now consider the cylinder $M \times I$ (Fig. 2), where $I=[0,1]$ is the unit interval. We may glue together the top and bottom of $M \times I$ [identifying $(x, 0)$ with $(\pi(x), 1)$ for any $x \in M]$ to make a closed manifold which we may call $\left(M \times S^{1}\right)_{\pi}$. (If $\pi$ is a gauge transformation, this procedure constructs a possibly nontrivial vector bundle over $M \times S^{1}$.)

Obviously, if $M$ happens to be $S^{n},\left(S^{n} \times S^{1}\right)_{\pi}$ and $S_{\pi}^{n+1}$ are closely related. One precise relation among them is the following. $\left(S^{n} \times S^{1}\right)_{\pi}$ is the connected sum of $S_{\pi}^{n+1}$ and $S^{n} \times S^{1}$. Here, the connected sum of two manifolds $A$ and $B$, denoted $A+B$, is obtained by cutting out an open ball from $A$ and one from $B$ and gluing $A$ and $B$ together along the boundary of the removed ball (Fig. 3). To show that $\left(S^{n} \times S^{1}\right)_{\pi}$ $\cong\left(S^{n} \times S^{1}\right)+S_{\pi}^{n+1}$, a picture may suffice (Fig. 4). It may be proved that $\lambda$ is additive under connected sums; that is $\lambda(A+B)=\lambda(A)+\lambda(B)$ for any $A$ and $B$. Hence $\lambda\left(\left(S^{n}\right.\right.$ $\left.\left.\times S^{1}\right)_{\pi}\right)=\lambda\left(S^{n} \times S^{1}\right)+\lambda\left(S_{\pi}^{n+1}\right)=\lambda\left(S_{\pi}^{n+1}\right)$, since it is easily shown that $\lambda\left(S^{n} \times S^{1}\right)=0$. To prove that $\lambda$ is additive under connected sums, let $\oplus$ be the disconnected sum, so $U \oplus V$ is a manifold with disconnected pieces $U$ and $V$. Obviously, $\lambda$ is additive 


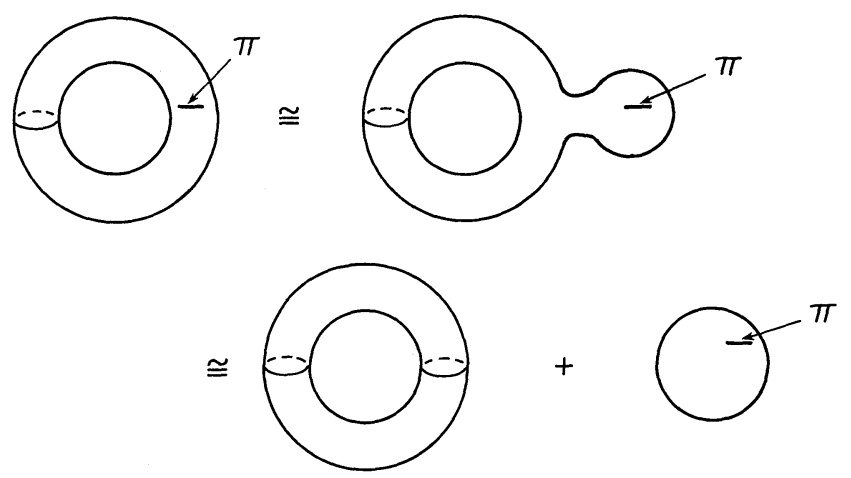

Fig. 4. The purpose of this figure is to show that $\left(S^{n} \times S^{1}\right)_{\pi} \cong\left(S^{n} \times S^{1}\right)+S_{\pi}^{n+1}$. The figure is drawn for $n=1$. $\left(S^{1} \times S^{1}\right)_{\pi}$ is a torus where an incision has been made and the two sides of the cut have been glued back together after transforming one side by $\pi$. This can be deformed into a form (second picture) that is obviously the connected sum of a standard torus $S^{1} \times S^{1}$ and a sphere in which a similar incision has been made and repaired; the latter is $S_{\pi}^{2}$

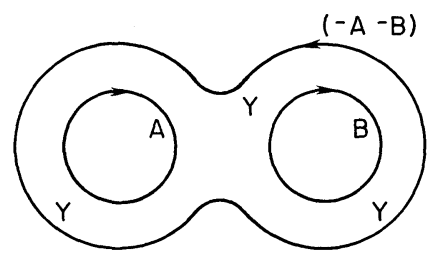

Fig. 5a and b. A construction that is useful for proving that various topological invariants are additive in connected sums. For any $\mathbf{A}$ and $\mathbf{B}, \mathbf{A}+\mathbf{B}+(-\mathbf{A}-\mathbf{B})$ is the boundary of a simple manifold $Y$. In the figure, $\mathbf{A}$ and $\mathbf{B}$ are circles. If $\mathbf{A}$ and $\mathbf{B}$ were two spheres, the picture would consist of two peas ( $\mathbf{A}$ and $\mathbf{B})$ in a pod ( $\mathbf{A}+\mathbf{B}$ with opposite orientation). The manifold $Y$ always has zero signature and admits a metric for which the Pontryagin densities are zero pointwise

under disconnected sums, since if $U$ bounds $X$ and $V$ bounds $Y, U \oplus V$ bounds $X \oplus Y$. For any oriented manifold $X$, let $(-X)$ be $X$ with opposite orientation. Now, let $Y$ be defined by $Y=A \oplus B \oplus(-A-B)$. Then $\lambda(Y)=\lambda(A)+\lambda(B)$ $-\lambda(A+B)$, since $\lambda$ is additive under disconnected sums and odd under change of orientation. To prove $\lambda$ is additive under connected sums we must show $\lambda(Y)=0$. But $Y$ bounds a manifold $Z$ that may be formed by a "peas in the pod" construction (Fig. 5). And $Z$ has $p_{1}^{3}=p_{1} p_{2}=\sigma=0$. [For instance, $\sigma$ depends only on the properties of closed six surfaces in $Z$ that cannot be deformed into surfaces in $Y$. There are no such. In addition, $Z$ admits a metric with $\left(\operatorname{Tr} R^{2}\right)^{3}=\left(\operatorname{Tr} R^{2}\right)\left(\operatorname{Tr} R^{4}\right)$ $=\operatorname{Tr} R^{6}=0$ at each point. These assertions are not hard to verify.]

We will eventually have need of a few more mathematical facts. The first such fact is that in $8 k+4$ dimensions the index of the Dirac or Rarita-Schwinger operator is always even. Indeed, let $C$ be the charge conjugation matrix, so $C^{-1} \Gamma_{\mu} C$ $=\Gamma_{\mu}^{*}$ for any gamma matrix $\Gamma_{\mu}$. Let $\mathscr{C}$ be the anti-unitary transformation $\mathscr{C} \psi=C \psi^{*}$. Then if $\psi$ is a zero mode of the Dirac or Rarita-Schwinger equation, so is $\mathscr{C} \psi$. Moreover [20], in $8 k+4$ dimensions $\mathscr{C}^{2}=-1$, so the number of zero 
modes is always even. (In $8 k$ dimensions, this is not true. The index of the RaritaSchwinger operator is one on the 8 dimensional spin manifold $H P^{2}$.)

The charge conjugation operator has another useful application. It is true [20] in $8 k+2$ dimensions (but not in $8 k+6$ ) that $\mathscr{C}^{2}=-1$. If $i \not D \psi=\lambda \psi$, then also $i \not D(\mathscr{C} \psi)$ $=\lambda(\mathscr{C} \psi) . \psi$ and $\mathscr{C} \psi$ must be linearly independent since $\mathscr{C}$ is an antiunitary operator with $\mathscr{C}^{2}=-1$. Now let $\Gamma=\Gamma_{1} \Gamma_{2} \ldots \Gamma_{8 k+2}$ be the chirality operator. The states $\bar{\Gamma} \psi$ and $\bar{\Gamma} \mathscr{C} \psi$ (which is $-\mathscr{C} \bar{\Gamma} \psi$, since $\mathscr{C} \bar{\Gamma}=-\bar{\Gamma} \mathscr{C}$ in $8 k+2$ dimensions) obey $i \not D(\bar{\Gamma} \psi)$ $=-\lambda \bar{\Gamma} \psi, i \not D(\bar{\Gamma} \mathscr{C} \psi)=-\lambda \bar{\Gamma} \mathscr{C} \psi$, since $\bar{\Gamma}$ anticommutes with $i \not D$. If $\lambda=0$ the pair of states $(\psi, \mathscr{C} \psi)$ may coincide with the pair $(\bar{\Gamma} \psi, \bar{\Gamma} \mathscr{C} \psi)$. For $\lambda \neq 0$ this is impossible, since one pair has Dirac eigenvalue $+\lambda$, and one pair has Dirac eigenvalue $-\lambda$. From these facts we may deduce the following. The number of zero eigenvalues of the Dirac operator in $8 k+2$ dimensions is always even, corresponding to pairs $(\psi, \mathscr{C} \psi)$. (Since $\psi$ and $\mathscr{C} \psi$ have opposite chirality, the Dirac index is always zero.) Now, if by varying the metric a pair of states $(\psi, \mathscr{C} \psi)$ migrates from non-zero eigenvalue $\lambda$ to $\lambda=0$, then the pair $(\bar{\Gamma} \psi, \bar{\Gamma} \mathscr{C} \psi)$ will migrate from $-\lambda$ to 0 at the same time. In this process, the number of zero eigenvalues changes by four. Since this and its inverse are the only possibly processes by which the number of zero eigenvalues can change, we see that in $8 k+2$ dimensions the number of zero modes of the Dirac operator is a topological invariant modulo four [21]. The discussion is unmodified if the fermions transform in a real representation of some gauge group, such as the adjoint representation of $\mathrm{O}(32)$ or $E_{8} \times E_{8}$. Note, however, that we have been counting states of both chiralities in the above discussion. If we are dealing with chiral fermions in $8 k+2$ dimensions, we keep only one state from each pair $(\psi, \mathscr{C} \psi)$. Hence, the number of zero modes of the chiral Dirac (or Rarita-Schwinger) operator in $8 k+2$ dimensions may be any integer, and is a topological invariant modulo 2.

The last facts we will need concern the signature of twelve dimensional spin manifolds with and without boundary. Analogous facts hold in four dimensions and we will consider the two cases in parallel. First, suppose $B$ has no boundary. In four dimensions, the signature and Dirac index are related by a well-known formula

$$
\frac{\sigma}{8}=\operatorname{index}(i \not D) \text {. }
$$

In twelve dimensions, there is no well-known formula analogous to (15). However, the "miraculous" anomaly cancellation found in [4] depended on a relation between $\sigma$, the Dirac index, and the Rarita-Schwinger index for twelve dimensional closed spin manifolds. The relation is

$$
\frac{\sigma}{8}=\operatorname{index}(R \cdot S)-3 \text { index }(i \not D)
$$

where index $(R \cdot S)$ is the index of the Rarita-Schwinger operator. Since we have shown that the Dirac and Rarita-Schwinger operators have an even index in $8 k+4$ dimensions, (15) and (16) show that $\sigma$ is divisible by sixteen for closed spin manifolds of four or twelve dimensions. (This is not so in $8 k$ dimensions; $H P^{2 k}$ is a spin manifold of dimension $8 k$ and of $\sigma=1$. It is true in $8 k+4$ dimensions for any $k$ [22].) 
Now, let $M$ be an eleven dimensional spin manifold. Suppose it can be bounded by the twelve dimensional spin manifold $B$. Then modulo sixteen, the signature of $B$ depends only on $M$, and not on $B$. For if $B$ and $B^{\prime}$ are two spin manifolds that bound $M$, then gluing them together along $M$ gives a closed twelve manifold $X$, whose signature must be divisible by sixteen. But $\sigma(X)=\sigma(B)-\sigma\left(B^{\prime}\right)$, so $\sigma(B)$ $=\sigma\left(B^{\prime}\right) \bmod 16$. The invariant just defined is additive under connected sums, by the same argument that shows this property for $\lambda$.

These considerations lead to an alternate proof that Milnor's eleven manifold $Q$ which bounds a spin manifold $B$ of signature eight and is homeomorphic to the standard sphere cannot be diffeomorphic to the standard sphere. For the standard sphere bounds a ball, which has $\sigma=0$, and $8 \neq 0 \bmod 16$.

\section{Instantons and Global Anomalies}

Our general analysis of global anomalies will be rather long and technical. Under certain conditions, however, global anomalies have a rather simple manifestation: they show up in the existence of an instanton field in which there are an odd number of fermion zero modes. Four dimensional theories with an odd number of zero modes in an instanton field always have global anomalies. This fact, which was originally found by computation [9], has an explanation due to Goldstone [23] which we will review later. In this section, we will show that in the ten dimensional anomaly free theories, the number of fermion zero modes in an instanton field is always even. This is a necessary but not sufficient condition for absence of global anomalies; there are theories (such as some $G_{2}$ theories recently considered by Harvey [12]), in which global anomalies are not related to having an odd number of zero modes in an instanton field. Since we will later discuss global anomalies in a general way, the discussion in this section is not strictly necessary. However, the arguments are so much simpler than those of Sects. IV and V that they seem worth describing separately. And the facts about ten dimensional instantons that we will need may have other applications. However, the rest of this paper can be read without reading this section.

This section will be organized as follows. First we will discuss which instantons are relevant. Then we will review Goldstone's argument showing that existence of an instanton with an odd number of zero modes always implies the existence of a global anomaly. Finally, we will show that instantons in anomaly free supersymmetric theories in ten dimensions always have an even number of fermi zero modes. This is trivial in the $N=2$ theories, which have an even number of Dirac and Rarita-Schwinger multiplets, so we will concentrate on the $N=1$ theories based on $\mathrm{O}(32)$ and $E_{8} \times E_{8}$.

In discussions of gravitational instantons, a basic fact about Yang-Mills instantons is sometimes overlooked. Let $I$ be a Yang-Mills instanton in a Euclidean space $M$ of dimension $d$. Then there is always an anti-instanton $\bar{I}$ with the property that $I+\bar{I}$ can be reached continuously from $A_{\mu}^{a}=0$. Here $I+\bar{I}$ is a pair consisting of an instanton and a widely separated anti-instanton.

Now, why do we have to include instantons in path integrals? Why can't we just declare that we only want to do integrals in the trivial topological sector? The answer, known [24] from early papers on instantons, is cluster decomposition. We 


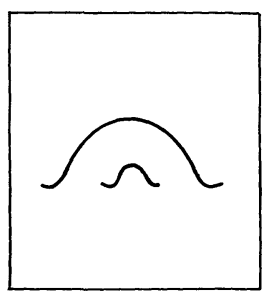

(a)

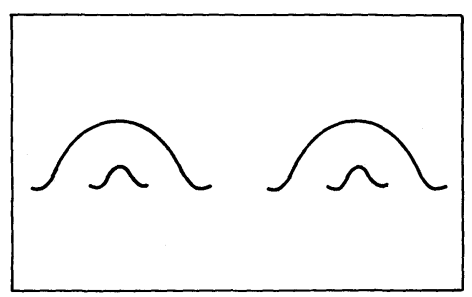

(b)

Fig. 6a and b. A handle on the plane, viewed as a gravitational instanton (Part a). There is no such thing as an anti-handle, though it is possible to imagine two widely separated handles (Part b)

must include $I+\bar{I}$ because it is in the topological class of $A_{\mu}^{a}=0$. If $x$ and $y$ are two points in $M$, this means one must include a field with an instanton near $x$ and an anti-instanton near $y$. If $y$ is very far from $x, I+\bar{I}$ produces, by cluster decomposition, the same effect that an isolated instanton at $x$ would have produced. This is why instantons play a role in Yang-Mills theory.

For many of the gravitational instantons that have been considered in the literature, this argument does not work. Consider an asymptotically Euclidean $n$ dimensional space with a localized topological defect, such as the handle of Fig. 6 , which we wish to regard as a gravitational instanton. Except for very special cases, there does not exist an anti-instanton $\bar{J}$ such that $J+\bar{J}$ is diffeomorphic to $R^{n}$. If $\bar{J}$ does not exist there is no need to include $J$ in path integrals.

Let us determine when $\bar{J}$ exists. If one of the Betti numbers $b_{i}$ of $J$ is non-zero for $1 \leqq i \leqq n-1$, then $\bar{J}$ cannot exist. For $b_{i}(J+\bar{J})=b_{i}(J)+b_{i}(\bar{J}) \geqq b_{i}(J)$ (since Betti numbers are non-negative). If $J+\bar{J} \cong R^{n}$, then $b_{i}(J+\bar{J})=0$ for $1 \leqq i \leqq n-1$, and we must have $b_{i}(J)=0$ for $1 \leqq i \leqq n-1$. Now let $X$ be the one point compactification of $J$ (defined by adding one point at infinity, as in the usual process of going from $R^{n}$ to $\left.S^{n}\right)$. For $n \geqq 4$, it follows from the Smale [13] $(n \geqq 5)$ and Freedman [25] $(n=4)$ proofs of the generalized Poincare conjecture that $X$ must be homeomorphic (but not necessarily diffeomorphic) to $S^{n}$ if $b_{i}(J)=0$ for $1 \leqq i \leqq n-1 .^{2}$

If $X$ is homeomorphic to $S^{n}$, does $\bar{J}$ exist? For $n=4$ this isn't known. We now focus on $n \geqq 5$. Here, if $X$ is homeomorphic to $S^{n}$, it must, as discussed in Sect. II, be $S_{n}^{n}$ for some diffeomorphism $\pi$ of $S^{n-1}$. Let $\pi^{-1}$ be the inverse diffeomorphism of $\pi$, and let $\bar{X}=S_{\pi^{-1}}^{n}$. Then $X+\bar{X}$ is diffeomorphic to $S^{n}$. This can be seen by a picture (Fig. 7). If $\bar{J}$ is related to $\bar{X}$ the way $J$ is related to $X$ (by removing a point and making the standard conformal change of metric to an asymptotically Euclidean space), then $\bar{J}$ is the desired inverse of $J$. Indeed, $J+\bar{J}$ is $R^{n}$ precisely because $X+\bar{X}$ is $S^{n}$.

It follows from this, in my opinion, that exotic spheres are the only gravitational instantons for which there is a sound basis within the presently understood framework of physics. When we discuss gravitational instantons, these are the ones we will discuss. Of course, it is always possible that future

2 If $n$ is 2 or $3, \bar{J}$ never exists unless $J=R^{n}$. For $n=2$ this follows from the classification of two manifolds, and for $n=3$ from the prime decomposition theorem for three manifolds [26] 


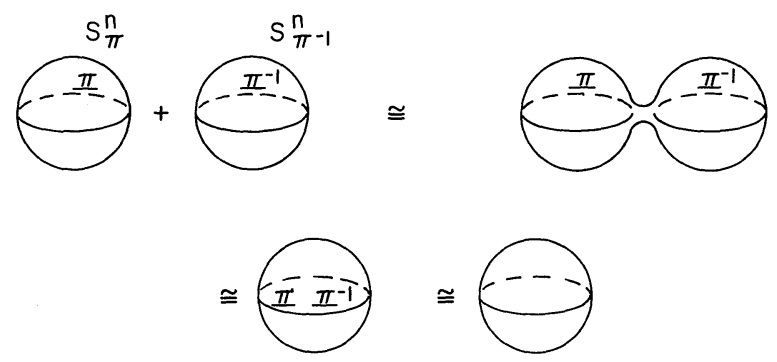

Fig. 7. This sequence of pictures is meant to show that $S_{n}^{n}+S_{\pi^{-1}}^{n} \cong S^{n}$

developments will give a sound physical basis for considering other gravitational instantons. This might even occur on the basis of string theory.

While this is outside our main line of development, let us pause to note that similar remarks can be made about gravitational solitons. In a theory in $n-1$ space and 1 time dimension, one could take an asymptotically Euclidean $n-1$ manifold $Y$ which is not diffeomorphic to $R^{n-1}$ and try to interpret it as a gravitational soliton. Does this make sense? It certainly makes sense and is correct if there is an asymptotically Euclidean manifold $\bar{Y}$ such that $Y+\bar{Y}$ is diffeomorphic to $R^{n-1}$. For then it is possible to make a continuous transition from $R^{n-1}$ to a state with a $Y$ particle and a far away $\bar{Y}$ antiparticle. In that case amplitudes such as $e^{+} e^{-} \rightarrow Y \bar{Y}$ occur unpreventably. If $\bar{Y}$ does not exist, the object $Y$ cannot be created or annihilated, and it is most natural to assume it is unphysical. Thus, in view of our previous remarks, at least for $n \geqq 6$, gravitational solitons correspond to exotic $n-1$ spheres. In standard general relativity we might expect such entities to collapse into black holes, but in view of the excellent short distance behavior of superstring theory, it would not be surprising if in that theory some of the seven [19] exotic nine spheres are realized as stable elementary particles.

Returning to gravitational instantons, the exotic spheres can be interpreted as tunneling events, just like Yang-Mills instantons. Let $g_{\mu \nu}$ be the Euclidean metric on $R^{n-1}$ in some coordinate system, and $g_{\mu \nu}^{\pi}$ its conjugate under $\pi$ (which, of course, is the Euclidean metric in the transformed coordinates). Now, consider the metric

$$
d s^{2}=d t^{2}+\left[(1-\lambda(t)) g_{\mu \nu}+\lambda(t) g_{\mu \nu}^{\pi}\right] d x^{\mu} d x^{\nu},
$$

where $t$ is "time" and $\lambda$ is a strictly monotonic function with $\lambda(-\infty)=0$, $\lambda(+\infty)=1$. The one point compactification of this space is the exotic sphere $S_{\pi}^{n}$. Thus, the instanton connected with $S_{\pi}^{n}$ is a tunneling event from $g$ to $g^{\pi}$. (This view of the instanton as a tunneling event makes it possible to see that, unlike other gravitational instantons, exotic spheres are present in the canonical, Hamiltonian, approach to quantum gravity.)

Now let us turn to the question of showing that if there are an odd number of fermion zero modes in an instanton field, there is always a global anomaly.

Yang-Mills instantons and exotic spheres are tunneling events associated with some gauge or coordinate transformation $\pi$ of the initial value surface. $\pi$ is realized in quantum field theory by some operator $\Lambda$. Gauss's law implies that gauge or coordinate transformations that can be reached continuously from the identity 
leave physical states invariant. $\Lambda$ is not under this restriction (if $\pi$ is topologically non-trivial), and in general $\Lambda=e^{i \theta}$, where $\theta$ is an analogue of $\theta_{\mathrm{QCD}}$.

Now, if the instanton associated with $\pi$ has an odd number of zero modes, this means in a Hamiltonian language that $\Lambda$ creates an odd number of fermions. Thus $(-1)^{F} \Lambda(-1)^{F}=-\Lambda$, where $(-1)^{F}$ is the operator that counts fermions mod2.

Now, following Goldstone, in an asymptotically Euclidean space let $J$ be the generator of a rotation about an arbitrary axis in space, and let

$$
G_{s}=\pi^{-1} \exp (-i s J) \pi \exp (i s J)
$$

for $0 \leqq s \leqq 2 \pi$. Obviously, $G_{0}=1$, and in a physical Hilbert space of states that obey Gauss's law, $G_{s}=1$ for all $s$, since $G_{s}$ is a gauge transformation that can be reached continuously from the identity. But $G_{2 \pi}=\pi^{-1}(-1)^{F} \pi(-1)^{F}=-1$. Since this contradicts the claim that $G_{s}=1$ for all $s$, it must be that the physical Hilbert space does not exist; this is the symptom of a global anomaly [9].

Now, let us verify that at least this kind of global anomaly does not arise in superstring theory, by showing that the number of fermion zero modes in an instanton field is always even. We must consider both gravitational and gauge instantons.

For gravitational instantons, we consider the five [19] exotic ten spheres. The $N=1$ supergravity theories have 497 elementary chiral Dirac fields (of various chirality) and one chiral Rarita-Schwinger field. We know from Sect. II that for either kind of field, the number of zero modes on any ten manifold $M$ is a topological invariant mod two. There are indeed [27] exotic ten spheres on which the chiral Dirac operator has an odd number of zero modes. To avoid trouble, we must show that, at least on exotic spheres, the chiral Rarita-Schwinger operator has an odd number of zero modes precisely when the Dirac one does.

For index purposes ${ }^{3}$, the chiral Rarita-Schwinger operator is equivalent to the equation $\not \psi_{\mu}=0$ (for a field $\psi_{\mu}$ of definite chirality; $\mu$ is a vector index) plus the contribution of three spin $1 / 2$ ghosts (of various chirality). The number of physical zero modes of the chiral Rarita-Schwinger equation, mod 2, equals the number of solution of $\not \psi_{\mu}=0$ minus the number of zero modes of the three chiral spin $1 / 2$ ghosts. Since three spin $1 / 2$ ghosts have, mod2, as many zero modes as 497 physical spin $1 / 2$ multiplets, what we must show is that on exotic 10 spheres, $\emptyset \psi_{\mu}=0$ always has an even number of solutions.

Let $\alpha$ and $\beta$ be, respectively, the number of solutions of the Dirac equation and of $D \psi_{\mu}=0$ on some exotic ten sphere. We will show $\beta=0 \bmod 2$ by showing $11 \alpha=\alpha$ $+\beta \bmod 2$. To show this, we arrange a chiral Dirac and a chiral Rarita-Schwinger field into one multiplet $\psi_{\alpha A}, \alpha=1 \ldots 16, A=1 \ldots 11$. Here $\alpha$ is a chiral spinor index, and $A$ is a vector index if $A=1 \ldots 10$, and a scalar index if $A=11$. The number of solutions of $D \psi_{A}=0$ is independent $\bmod 2$ is what real connection is used on the $A$ index. If the Riemannian connection is used, the number of zero modes is $\alpha+\beta$. However, a theorem of Kervaire and Milnor [19] ensures that it is possible to choose a real connection on the $A$ index so that the equation $D \psi_{A}=0$ is equivalent

3 This is only true for index purposes because the actual gauge fixed Rarita-Schwinger equation involves non-minimal terms. (Index purposes include the mod 2 index being computed here) 
(up to a gauge transformation) to eleven decoupled Dirac equations. With this connection the number of zero modes is $11 \alpha$, so this shows $11 \alpha=\alpha+\beta \bmod 2$.

The theorem of Kervaire and Milnor that is relevant is their statement that exotic spheres are $s$-parallelizable. This means that if $T$ is the tangent bundle of an exotic sphere and $\varepsilon$ is a trivial line bundle, then $T \oplus \varepsilon$ is topologically trivial. In other words, the theorem says roughly that on an exotic sphere, a vector field and a scalar field can topologically be rearranged into eleven scalar fields. The proof of this theorem in $8 k+2$ dimensions is somewhat subtle.

This completes our discussion of gravitational instantons; we turn to YangMills instantons. Since $\pi_{9}(\mathrm{O}(32))=Z_{2}, \pi_{9}\left(E_{8} \times E_{8}\right)=0$, the interesting case is $\mathrm{O}(32)$, and there is precisely one $\mathrm{O}(32)$ instanton to be considered.

Our first task will be to give an explicit description of the $\mathrm{O}(32)$ instanton on $S^{10}$, which we take with standard metric. The spin connection on $S^{10}$ is an $\mathrm{O}(10)$ gauge field. Let us consider the $\mathrm{O}(10)$ embedding in $\mathrm{O}(32)$ defined by saying that the vector representation of $\mathrm{O}(32)$ transforms as the real, 32 dimensional spinor representation of $\mathrm{O}(10)$. This embedding of $\mathrm{O}(10)$ in $\mathrm{O}(32)$ turns the spin connection of $S^{10}$ into an $\mathrm{O}(32)$ gauge field - which turns out to be the instanton.

To show that it is the instanton, it is enough to show that it is topologically non-trivial - since the instanton is the only topologically non-trivial class of $\mathrm{O}(32)$ gauge fields on $S^{10}$. To show it is topologically non-trivial, it is enough to show that the (non-chiral) Dirac equation on $S^{10}$ with this gauge field, in some representation, has a non-zero number of zero eigenvalues mod4. The vector representation is a good choice.

Consider a spinor $\psi_{\alpha x}$, where $\alpha$ is a spinor index on $S^{10}$ and $x$ labels the vector of $\mathrm{O}(32)$. In fact, $x$ is, in view of the construction above, equivalent to a spinor index of $\mathrm{O}(10)$. The Dirac equation for spinors with an extra spinor index is equivalent to the equation $\left(d+d^{*}\right) \phi=0$ for differential forms $\phi$. The number of zero modes is the sum of the Betti numbers. For $S^{10}, b_{0}=b_{10}=1$ and the others vanish. So there are two zero modes. Since $2 \neq 0 \bmod 4$, this field is topologically non-trivial and is the instanton.

Now we turn to the representation which actually is relevant for ten dimensional supergravity and superstring theory, which is the adjoint representation. Thus, we need to count the number of zero modes mod 4 of a chiral field $\psi_{\alpha x y}$ $=-\psi_{\text {ayx }}$. Since $x$ and $y$ are equivalent to spinor indices of $\mathrm{O}(10)$, this field really has three $\mathrm{O}(10)$ spinor indices. Now, zero modes of the $\psi_{\text {axy }}$ field will form representations of the $\mathrm{O}(11)$ rotation group of $S^{10}$ (since the spin connection, which entered this construction, is certainly $\mathrm{O}(11)$ invariant). The representations that will appear are spin representations of $O(11)$ (which change sign under a $2 \pi$ rotation in $\mathrm{O}(11)$ ), since $\psi_{\alpha x y}$ has three spinor indices, an odd number. All the spin representations of $\mathrm{O}(11)$ have dimension divisible by 32 , so the chiral $\psi_{\text {axy }}$ field certainly has an even number of zero modes.

This completes the demonstration that the number of zero modes in an instanton field is always even in the $\mathrm{O}(32)$ and $E_{8} \times E_{8}$ theories.

Let us conclude this section with some remarks about the canonical or Hamiltonian treatment of global gravitational anomalies. The Euclidean treatment of global gauge anomalies involves a global gauge transformation $U\left(t, x^{i}\right)$ in space-time ( $t$ is time and $x^{i}$ are space coordinates). The Hamiltonian treatment [9] 
involves instead a one parameter family of spatial gauge transformation such as the family $G_{s}$ in Eq. (18). The connection between them is trivial because $U\left(t, x^{i}\right)$ may be viewed as a one parameter family $U_{t}\left(x^{i}\right)$ of spatial gauge transformations.

In general relativity the situation is puzzling at first because there is no obvious connection between a diffeomorphism in $d$ dimensions $\left(t, x^{i}\right) \rightarrow\left(t^{\prime}\left(t, x^{i}\right), x^{\prime j}\left(t, x^{i}\right)\right)$ and a one parameter family of diffeomorphisms of $d-1$ dimensions $x^{i} \rightarrow x_{t}^{\prime j}\left(x^{i}\right)$. The connection between them is made by a highly non-trivial theorem due to Cerf [15]. Cerf proved that in five or more dimensions every diffeomorphism of $d$ space which vanishes at infinity is deformable topologically to a diffeomorphism that leaves the time invariant $\left(t, x^{i}\right) \rightarrow\left(t, x^{j}\left(t, x^{i}\right)\right)$. Such a diffeomorphism is indeed a one parameter family ( $t$ is the parameter) of diffeomorphisms of $d-1$ space. This theorem makes possible a Hamiltonian treatment of global gravitational anomalies just analogous to the Hamiltonian treatment of global gauge anomalies.

\section{A General Formula for Global Anomalies}

In this section we will develop a formula that is useful for analyzing global anomalies. We will formulate the problem in terms of global gravitational anomalies, but similar reasoning applies to the gauge case.

Consider a quantum field theory formulated on some (Euclidean signature) manifold $M$. Let $\pi$ be a diffeomorphism of $M$. Let $I\left(g_{\mu \nu}\right)$ be the effective action of our theory, as a functional of the metric $g_{\mu \nu}$ of $M$. Let $g_{\mu \nu}$ transform into $g_{\mu \nu}^{\pi}$ under $\pi$. If $\Delta I=I\left(g_{\mu \nu}^{\pi}\right)-I\left(g_{\mu \nu}\right)$ is not an integer multiple of $2 \pi i$, the theory suffers from global anomalies.

To define $\Delta I$ precisely requires a little bit of care. Let $g_{\mu v}^{t}, 0 \leqq t \leqq 1$, be a one parameter family of metrics interpolating from $g_{\mu \nu}$ to $g_{\mu \nu}^{\pi}$. For instance, we may take $g_{\mu \nu}^{t}=(1-t) g_{\mu \nu}+t g_{\mu \nu}^{\pi}$. Then we define

$$
\Delta I=\int_{0}^{1} d t \frac{d}{d t} I\left(g_{\mu v}^{t}\right)
$$

and this is the quantity we must study.

We will concentrate on problems in which the one loop determinants are complex. Real determinants can at worst change in sign under $\pi$, and the sign can be studied as in [9]. A useful simplification for complex determinants is that a complex function will generically not vanish as a function of the one real variable $t$, and we will assume that our determinants are non-zero for all $t, 0 \leqq t \leqq 1$.

In studying $\Delta I$, we will first consider the case when the one loop determinant of the matter fields (summing over all multiplets) is free of perturbative anomalies. After treating this case, we will study the more subtle situation in which one loop matter anomalies are cancelled by the local terms introduced by Green and Schwarz.

As a typical case, we consider first the anomaly due to a spin $1 / 2$ Weyl fermion. For a Majorana-Weyl fermion (possible in $8 k+2$ dimensions), the effective action is $I=1 / 2 \ln \operatorname{det} i \not_{R}$; here $\not_{R}$ is the Dirac operator restricted to fermi fields that obey $\left(\frac{1+i \bar{\Gamma}}{2}\right) \psi=\psi$, with $\bar{\Gamma}=i \Gamma^{1} \Gamma^{2} \ldots \Gamma^{2}$ being the chirality projection operator 
$\left(\bar{\Gamma}^{2}=1\right)$. Under an arbitrary change in the Dirac operator, $\not D \Rightarrow \not D+\delta \not$, the change in the action is

$$
\delta I=\frac{1}{2} \operatorname{Tr} \frac{1}{i \not D} \delta i \not D\left(\frac{1+i \bar{\Gamma}}{2}\right),
$$

where we have chosen to exhibit explicitly the chirality projection operator $\left(\frac{1+\bar{\Gamma}}{2}\right)$, so that now $i \not D$ is the full-fledged, parity-conserving Dirac operator.

In (19), the parity-conserving term $\frac{1}{4} \operatorname{Tr} \frac{1}{i \not D} \delta \not \not D=\delta\left((\operatorname{det} i \not D)^{1 / 4}\right)$ may be dropped. The reason for this is that $(\operatorname{det} i \not D)$ is parity conserving and positive, and $(\operatorname{det} i \not D)^{1 / 4}$ would change in phase, as a function of $t$, only when det $i \not D$ vanishes. We have already noted that in $4 k+2$ dimensions, for a generic interpolation from $g_{\mu \nu}$ to $g_{\mu \nu}^{\pi}$, the Dirac determinant has no zeros. (The real determinant det $i \not D$ has a factorization $\operatorname{det} i \not D=\operatorname{det} i \not_{L} \cdot \operatorname{det} i \not D_{R}$, where $\operatorname{det} i \not_{L}$ and $\operatorname{det} i \not D_{R}$ are complex functions that do not generically vanish as a function of one real variable.) If one chooses to consider a non-generic interpolation from $g_{\mu \nu}$ to $g_{\mu \nu}^{\pi}$ for which det $i \not D$ has zeros, the parity-conserving part of (18) cannot be dropped, and a compensating subtlety would appear in analyzing the parity violating part. ${ }^{4}$

We may hence reduce (19) to

$$
\delta I=\frac{i}{4} \operatorname{Tr} \frac{1}{i \not D} \delta i \not D \bar{\Gamma}=\frac{i}{4} \sum_{j}\left(\frac{1}{\lambda_{j}}-\frac{1}{\lambda_{j}+i M}\right)\left\langle\psi_{j}|\delta i \not D \bar{\Gamma}| \psi_{j}\right\rangle
$$

where $\psi_{j}$ and $\lambda_{j}$ are eigenstates and eigenvalues of the Dirac operator (which depend on $t$ ), and where we have taken care to include the contribution of a PauliVillars regulator field of mass $M$. However, in and only in theories that are free of perturbative anomalies, the contribution of the regulator fields vanishes. The reason for this is as follows. The contribution of the massive regulator fields in (20) is a local functional of the metric tensor. This functional, if not zero, is never generally covariant since there is simply no generally covariant functional of the metric with the right dimension and quantum numbers. It is precisely from this functional that perturbative anomalies are extracted; the connection has recently been discussed in detail [28]. The induced functional due to the massive regulators hence vanishes precisely when the matter determinants, after summing over all matter multiplets, are free of perturbative anomalies. When anomaly cancellation depends on the local terms introduced by Green and Schwarz, the regulator contribution does not vanish and must be included.

4 Identically, a zero of det $i \not D$ in $8 k+2$ dimensions is typically a fourth order zero, since the number of zero modes of the Dirac operator is a topological invariant $\bmod 4$ in $8 k+2$ dimensions. In passing through such a zero, $(\operatorname{det} i \not D)^{1 / 4}$ would change sign. In $8 k+6$ dimensions, one always has complex chiral fermions, so the object encountered is $(\operatorname{det} i \not D)^{1 / 2}$. In $8 k+6$ dimensions, the number of zero modes of the Dirac operator is only invariant mod2, so det $i \not D$ has double zeros at which $(\operatorname{det} i \not D)^{1 / 2}$ changes sign. In either case such zeros do not generally appear as a function of one real variable 
From (20), we see that when the regulator contribution is absent, the total change in the action between $t=0$ and $t=1$, after summing over all multiplets $Q$, is

$$
\Delta I=\frac{i}{4} \sum_{Q} \sum_{j} \int_{0}^{1} d t \frac{1}{\lambda_{j}(t)}\left\langle\psi_{j}(t)\left|\left(\frac{d i \not D}{d t}\right) \bar{\Gamma}\right| \psi_{j}(t)\right\rangle .
$$

We must reduce (21) to a manageable expression.

Equation (21) involves eigenstates of the Dirac operator on a manifold $M$ of dimension $n=4 k+2$. The right-hand side of (21) is expected to depend only on $M$ and the diffeomorphism $\pi$, not on the interpolation $g_{\mu \nu}^{t}$, though at this point that is not obvious. Usually, the only simple way to study a diffeomorphism $\pi$ is to investigate the associated manifold $\left(M \times S^{1}\right)_{\pi}$ discussed in Sect. II. The simplest properties of $\left(M \times S^{1}\right)_{\pi}$ are invariants of a manifold $B$ which has it for boundary. The only evident connection between $\left(M \times S^{1}\right)_{\pi}$ and $B$ in which spinors play a role is the Atiyah-Patodi-Singer theorem concerning the $\eta$-invariant [29]. The $\eta$ invariant can be defined as

$$
\eta=\lim _{\varepsilon \rightarrow 0} \sum_{E_{A} \neq 0}\left(\operatorname{sign} E_{A}\right) \exp -\varepsilon\left|E_{A}\right|,
$$

where $E_{A}$ are the eigenvalues of the Dirac operator on $\left(M \times S^{1}\right)_{\pi}$. The AtiyahPatodi-Singer theorem asserts (for the spin 1/2 case) that

$$
\frac{\eta}{2}=\operatorname{index}_{B}(i \not D)-\int_{B} \hat{A}(R),
$$

where $\operatorname{index}_{B}(\not D D)$ is the index of the Dirac operator on $B$ and $\hat{A}(R)$ is the polynomial in the curvature tensor $R$ that enters the index theorem for manifolds without boundary. (Thus, if $B$ has no boundary, $\operatorname{index}_{B}(i \not D)=\int_{B} \hat{A}(R)$.) $\hat{A}(R)$ is also the $\frac{n+2}{2}$ form that is related, formally, to perturbative anomalies in $n$ dimensions. A very natural strategy is to try to relate (21) to the $\eta$ functional of $\left(M \times S^{1}\right)_{\pi}$

On $\left(M \times S^{1}\right)_{\pi}$ we take the metric tensor

$$
d s^{2}=d t^{2}+\varepsilon g_{\mu \nu}^{t}\left(x^{\alpha}\right) d x^{\mu} d x^{\nu}
$$

where $x^{\mu}$ are coordinates on $M$, and $\varepsilon$ is a real number chosen so that the Dirac equation on $\left(M \times S^{1}\right)_{\pi}$ can be solved adiabatically in $t$. This is so if $\varepsilon$ is small enough so that the Dirac operator on $M$ with metric $\varepsilon g_{\mu \nu}$ has widely enough separated eigenvalues. By absorbing $\varepsilon$ in the normalization of $g_{\mu \nu}^{t}$ (which has hitherto been arbitrary) we may assume that this is true at $\varepsilon=1$. It should be pointed out that the simplicity of (24) is deceptive since $\left(x^{\mu}, t=0\right)$ is to be identified with $\left(\pi\left(x^{\mu}\right), t=1\right)$, where $\pi$ is highly non-trivial.

To formulate the Dirac equation on $\left(M \times S^{1}\right)_{\pi}$ we need an extra gamma matrix in addition to the gamma matrices $\Gamma^{1}, \Gamma^{2}, \ldots, \Gamma^{n}$ of $M$. We can take it to be $\bar{\Gamma}$, so that the Dirac equation in $\left(M \times S^{1}\right)_{\pi}$ is

$$
\left(i \bar{\Gamma} \frac{d}{d t}+i \not D^{t}\right) \Psi=E \Psi
$$


This equation will be solved in an adiabatic approximation, in terms of the eigenstates $\psi_{n}^{t}$ and eigenvalues $\lambda_{n}^{t}$ of the hermitian operator $i \not D^{t}$.

To solve the Schrödinger equation in an adiabatic approximation, one usually picks a time dependent eigenstate of $H, \psi_{0}(t)$, and writes $\Psi(t)=A(t) \psi_{0}(t)$ (the $x^{\alpha}$ dependence is being suppressed). In the case at hand, by writing (25) in the Schrödinger-like form $i \frac{d \Psi}{d t}=X \psi$ with $X=(E \bar{\Gamma}-i \bar{\Gamma} \not D)$, we see that the analogue is to work with an eigenstate of $X$. If $\psi_{0}$ is an eigenstate of $i \not D$, so is $\tilde{\psi}_{0}=\bar{\Gamma} \psi_{0}$ (with opposite eigenvalue; if $i \not D \psi_{0}=\lambda_{0} \psi_{0}$, then $\left.i \not D \tilde{\psi}_{0}=-\lambda_{0} \tilde{\psi}_{0}\right)$. Eigenvalues of $X$ can be found as linear combinations of $\psi_{0}$ and $\tilde{\psi}_{0}$. Instead of explicitly diagonalizing $X$, it is easier to write $\Psi(t)=A(t) \psi_{0}+B(t) \tilde{\psi}_{0}$ and solve for $A$ and $B$.

In studying adiabatic evolution in quantum mechanics, it is convenient to fix the time dependence of the phase of the states by requiring $\left\langle\psi_{0} \mid \frac{d \psi_{0}}{d t}\right\rangle=0$. In the case at hand this implies $\left\langle\tilde{\psi}_{0} \mid \frac{d \tilde{\psi}_{0}}{d t}\right\rangle=0$. We must note that $\psi_{0}(t=1)$ is to be related to $\psi_{0}(t=0)$ by means of the diffeomorphism $\pi$. In general we might find $\psi_{0}^{\pi}(t=1)=e^{i \alpha} \psi_{0}(t=0)$ for some $\alpha$ (where $\psi_{0}^{\pi}(t=1)$ is the transform of $\psi_{0}(t=1)$ by $\left.\pi\right)$. This would imply $\tilde{\psi}_{0}^{\pi}(t=1)=e^{i \alpha} \tilde{\psi}_{0}(t=0)$. This would imply for $A$ and $B$ the boundary condition $A(1)=e^{i \alpha} A(0), B(1)=e^{-i \alpha} B(0)$. We will see that the value of $\alpha$ does not matter.

The easiest way to find the right equation for $A$ and $B$ is probably to note that the Dirac equation (25) is deducible from $\delta L=0$, where $L=\int d t\langle\psi|\left(i \bar{\Gamma} \frac{d}{d t}+i \not D_{t}\right.$ $-E)|\Psi\rangle$. Inserting the ansatz $\Psi=A(t) \psi_{0}(t)+B(t) \tilde{\psi}_{0}(t)$, and varying with respect to $A$ and $B$, one gets the equation

$$
U \phi=E \phi
$$

where

$$
U=i \sigma_{1} \frac{d}{d t}+\lambda_{0}(t) \sigma_{3}+\mu_{0}(t)
$$

Here $\sigma_{1}$ and $\sigma_{3}$ are Pauli spin matrices,

and

$$
\phi(t)=\left(\begin{array}{c}
A(t) \\
B(t)
\end{array}\right)
$$

$$
\mu_{0}(t)=\left\langle\psi_{0}|\bar{\Gamma}| i \frac{d \psi_{0}}{d t}\right\rangle=\left\langle\tilde{\psi}_{0}|\bar{\Gamma}| i \frac{d \tilde{\psi}_{0}}{d t}\right\rangle
$$

It is useful to define

$$
v_{0}=\int_{0}^{1} d t \mu_{0}(t)
$$


and

$$
\mu_{0}(t)=v_{0}+\frac{d \beta}{d t}
$$

where $\beta(t)$ is some function that obeys $\beta(1)=\beta(0)$. Now, we would like to calculate the $\eta$ invariant of $U$, defined by

$$
\eta_{U}=\lim _{\varepsilon \rightarrow 0} \sum_{E} \operatorname{sign} E \cdot \exp -\varepsilon|E|,
$$

where $E$ ranges over the eigenvalues of $U$. If $\lambda_{0}(\mathrm{t})=0, U$ can be exactly diagonalized. The eigenfunctions are

$$
\psi_{n}^{ \pm}=\exp (-2 \pi \operatorname{int} \pm i \beta(t))\left(\begin{array}{c}
1 \\
\pm 1
\end{array}\right)
$$

with energies

$$
E_{n}^{ \pm}= \pm 2 \pi n+v_{0}
$$

Inserting (34) in (32) gives

$$
\eta_{U}=\frac{2}{\pi} v_{0} \bmod 2
$$

(The "mod2" is needed if $\left|v_{0}\right|>\pi$.) If $\lambda_{0}(t) \neq 0$, (34) is no longer exact. But (32) shows that $\eta_{U}$ only depends on the asymptotic behavior of the eigenvalues for large $E$. Even if $\lambda_{0}(t) \neq 0$, the $\lambda_{0}(t) \sigma_{3}$ term in (27) only shifts the $E_{n}$ by an amount of order $1 / n$ (since $\sigma_{3}$, acting on a state $\psi_{n}^{ \pm}$of unperturbed energy $\pm 2 \pi n+v_{0}$ gives a state $\psi_{n}^{\mp}$ of unperturbed energy $\mp 2 \pi n+v_{0}$; the energy denominator is hence of order $n$ for large $n$ ). Such asymptotically small shifts do not modify $\eta$, so (35) is exact for any $\lambda_{0}(t)$. (More elegant derivations of (35) have been given by S. DellaPietra, who also corrected an error in may original treatment, and A. Niemi.)

Now we need a technical aside whose sole purpose is to turn the mod 2 in (35) into mod4. [This will be essential for the $\mathrm{O}(32)$ and $E_{8} \times E_{8}$ theories.] We recall from Sect. II that in $8 k+2$ dimensions, there is an antiunitary operator $\mathscr{C}$ obeying $\mathscr{C}^{2}=-1$ and $[\mathscr{C}, i \not D]=0$. Apart from the pair of states $\psi_{0}, \tilde{\psi}_{0}$, we could have performed the same calculation with the states $\psi_{1}=\mathscr{C} \psi_{0}, \tilde{\psi}_{1}=\bar{\Gamma} \psi_{1}$. Since $\left\langle\psi_{1}|\bar{\Gamma}| \frac{d \psi_{1}}{d t}\right\rangle=\left\langle\psi_{0}|\bar{\Gamma}| \frac{d \psi_{0}}{d t}\right\rangle$, the sector $\left(\psi_{1}, \tilde{\psi}_{1}\right)$ makes the same contribution as the sector $\left(\psi_{0}, \tilde{\psi}_{0}\right)$ in the above calculation. So if we refer to $\eta$ in the new sector as $n_{\mathscr{C U}}$, then

$$
n_{U}+\mathrm{n}_{\mathscr{C} U}=\frac{4}{\pi} v_{0} \bmod 4
$$

In $8 k+6$ dimensions an analogous doubling of $\eta$ occurs for a more trivial reason. The basic object in $8 k+6$ dimensions is a complex chiral field, so all our formulas must be doubled. 
To relate our result for $\eta_{U}$ to the old formula (21) for $\Delta I$, let us re-examine definition (27) of $\mu_{0}(t)$. According to quantum mechanical perturbation theory,

$$
\frac{d \psi_{0}}{d t}=\sum_{j \neq 0} \frac{1}{\lambda_{0}-\lambda_{j}}\left|\psi_{j}\right\rangle\left\langle\psi_{j}\left|\left(\frac{d}{d t} i \not D\right)\right| \psi_{0}\right\rangle .
$$

Hence

$$
\mu_{0}(t)=\left\langle\psi_{0}|\bar{\Gamma}| i \frac{d \psi_{0}}{d t}\right\rangle=\sum_{j \neq 0}\left\langle\psi_{0}|\bar{\Gamma}| \psi_{j}\right\rangle \frac{1}{\lambda_{0}-\lambda_{j}}\left\langle\psi_{j}\left|\left(\frac{d}{d t} i \not D\right)\right| \psi_{0}\right\rangle .
$$

But

$$
\left\langle\psi_{0}|\bar{\Gamma}| \psi_{j}\right\rangle \neq 0 \quad \text { only if } \quad \psi_{j}=\tilde{\psi}_{0}=\bar{\Gamma} \psi_{0}
$$

so

$$
\mu_{0}(t)=\frac{1}{2 \lambda_{0}}\left\langle\psi_{0}\left|\bar{\Gamma} \frac{d}{d t}(i \not D)\right| \psi_{0}\right\rangle
$$

and hence

$$
v_{0}=\int_{0}^{1} d t \frac{1}{2 \lambda_{0}}\left\langle\psi_{0}\left|\bar{\Gamma} \frac{d}{d t}(i \not D)\right| \psi_{0}\right\rangle .
$$

Combining (21) and (40), we see

$$
\Delta I=i \sum_{\text {pairs }} v_{0}(\text { Pair })=2 i \sum_{\text {fours }} v_{0}(\text { Four }),
$$

where the sum runs over a pair of state $\left(\psi_{0}, \tilde{\psi}_{0}\right)$ or a foursome of states $\left(\psi_{0}, \tilde{\psi}_{0}, \psi_{1}\right.$, $\tilde{\psi}_{1}$ ). [In going from (21) to (41), one must remember that (21) was written as a sum over states, not a sum over pairs or foursomes of states.] Looking back to (35), we see $v_{0}($ Four $)=\frac{\pi}{4}\left(\eta_{U}+n_{\mathscr{C} U}\right) \bmod \pi$, so

$$
\Delta I=\frac{\pi i}{2} \sum_{\text {Fours }}\left(\eta_{U}+\eta_{\mathscr{C U}}\right) \bmod 2 \pi i .
$$

Finally we may drop the " $\bmod 2 \pi i$ " since we only want to know $\Delta I \bmod 2 \pi i$. Also, $\sum_{\text {Fours }}\left(\eta_{U}+\eta_{\mathscr{C} U}\right)=\eta$, where $\eta$ is the $\eta$ invariant of the full-fledged Dirac operator on $\left(M \times S^{1}\right)_{\pi}$. Hence $(\bmod 2 \pi i)$

$$
(\Delta I)_{\text {Dirac }}=\frac{\pi i}{2} \eta_{D},
$$

where $\eta_{D}$ is the Dirac $\eta$ invariant on $\left(M \times S^{1}\right)_{\pi}$. For Dirac fields in $8 k+2$ dimensions, this is the end of the story. For Rarita-Schwinger and self-dual antisymmetric tensor fiels, there are some additional technicalities, however.

The Rarita-Schwinger operator on $M$ has a vector index with $8 k+2$ components, while on $\left(M \times S^{1}\right)_{\pi}$ the vector index has $8 k+3$ components. The extra component is a scalar as seen on $M$. To carry out the construction above we have to consider on $M$ a multiplet consisting of a Rarita-Schwinger field and a Dirac field. They combine nicely into the Rarita-Schwinger field on $\left(M \times S^{1}\right)_{\pi}$, and the above discussion goes through, giving $(\Delta I)_{\text {Dirac }}+(\Delta I)_{\mathrm{R} . \mathrm{s} .}=\frac{\pi i}{2} \eta_{R}$, where $\eta_{R}$ is the 
Rarita-Schwinger $\eta$ invariant on $\left(M \times S^{1}\right)_{\pi}$. In other words,

$$
(\Delta I)_{\mathrm{R} . \mathrm{S} .}=\frac{\pi i}{2}\left(\eta_{R}-\eta_{D}\right) .
$$

For self-dual tensor fields matters are trickier, unfortunately. First of all, there is not a convenient Lagranigan for this field. For that reason, in [4] the normalization of the anomaly for this field was found by examining Feynman diagrams; this gave an unexpected numerical factor (see footnote 6 of that paper). To try to normalize the contribution to the global anomaly in this way would be tedious, to say the least. For this reason, we will here follow another method [4], which is very efficient and almost certainly correct but not quite rigorous (because the connection between the effective action of the self-dual tensor and the determinant of the operator we are about to introduce has never been deduced from first principles).

Consider in $8 k+2$ dimensions a field $\phi_{\alpha \beta}$ where $\alpha$ is a chiral spinor index and $\beta$ is a spinor index that may have both chiralities. Actually, $\phi$ is equivalent to a certain collection of antisymmetric tensors. Let $Q$ be the chiral Dirac operator acting on the $\alpha$ index; that is, $(Q \phi)_{\alpha^{\prime} \beta}=D_{\alpha^{\prime} \alpha} \phi_{\alpha \beta}$. The equation $Q \phi=0$ is easily studied in Minkowski space. It describes propagation of two real self-dual antisymmetric tensors (one comes from each chirality of $\beta$ ) and various anomaly free fields. It is thus highly plausible that for the sake of computing anomalies, the effective action of one real self-dual tensor can be taken to be $-1 / 4 \ln \operatorname{det} Q$ (the sign differs from the Dirac case because of opposite statistics).

The change of $\ln \operatorname{det} Q$ in interpolating from a metric $g$ to $g^{\pi}$ can be computed as we have done for the Dirac operator. Indeed, $Q$ is just a Dirac operator acting on a field with an extra index. Hence the change of $-1 / 4 \ln \operatorname{det} Q$ is $-i \pi / 4 \eta_{S}$; here $\eta_{S}$ is the eta invariant of an operator $S$ that was introduced in [29]. It is the operator *d acting on the even degree differential forms on $\left(M \times S^{1}\right)_{\pi}$. [The restriction to even or equivalently to odd degree arises because a field $\phi_{\alpha \beta}$ with two non-chiral spinor indices $\alpha$ and $\beta$ is equivalent to the whole de Rham complex of $M$, but to only half the de Rham complex of $\left(M \times S^{1}\right)_{\pi}$.] So we arrive at the result

$$
\Delta I_{S}=-\frac{\pi \mathrm{i}}{4} \eta_{S}
$$

This result must be treated with a certain degree of caution. First, we have already noted that use of the operator $Q$ is not quite rigorous. Second, and perhaps more essential, the operator $Q$ will have zero modes, and this raises the question of what we mean by $\operatorname{det} Q$, which appeared at intermediate stages of the discussion.

If $M$ has dimension $8 k+2$, the self dual tensor on $M$ is a $4 k+1$ form. If the $4 k+1$ Betti number of $M, b_{4 k+1}(M)$ is non-zero, then the self-dual tensor has zero modes on $M$, and its effective action on the compact space $M$ could be treated only with much more care than we have given. We hence assume that $b_{4 k+1}(M)=0 .{ }^{5}$

5 In case $b_{4 k+1} \neq 0$, I believe the contribution of the self-dual tensor to a possible global anomaly depends on the action of $\pi$ on $H^{4 k+1}(M ; R)$. Consideration of this point would carry us far afield. However, the analogous question arises in connection with modular invariance in string theory 
Even so, other spin fields descirbed by $Q$ have zero modes [since, for instance, $\left.b_{0}(M)=1\right]$, and this means our manipulations involving $\operatorname{det} Q$ were naive.

As long as $b_{4 k+1}(M)=0$, the zero modes of $Q$ are probably just a technicality. They arise for wrong spin components of $\phi_{\alpha \beta}$ that were introduced just for convenience and have no anomalies. Dividing $\operatorname{det} Q$ by the determinant of a suitable operator that describes the wrong spin fields (for instance, the KleinGordon operator for scalars), one should be able to eliminate the unwanted zeros of $\operatorname{det} Q$. Our starting point - the self-dual tensor effective action - is well-defined as long as $b_{4 k+1}(M)=0$, and the answer (45) is also well-defined and presumably correct.

In sum, for a theory with $N_{D}, N_{R}$, and $N_{S}$ chiral Dirac, Rarita-Schwinger, and self-dual tensor fields, the change in the effective action under a diffeomorphism is

$$
\Delta I=\frac{\pi i}{2}\left(N_{D} \eta_{D}+N_{R}\left(\eta_{R}-\eta_{D}\right)-\frac{N_{S}}{2} \eta_{S}\right)
$$

This is the answer to our problem only in a very limited way. To calculate $\eta_{D}, \eta_{R}$, and $\eta_{S}$ explicitly would hardly be feasible. From (46) it is not even obvious that $\Delta I$ is a topological invariant (i.e., depends only on the topology of $M$ and the topological class of $\pi$ ). Happily, the Atiyah-Patodi-Singer theorem gives a practical method to evaluate (46) in terms of properties of a spin manifold $B$ whose boundary is $\left(M \times S^{1}\right)_{\pi}$, if such $B$ exists. Assuming $B$ exists, the theorem asserts that

$$
\begin{gathered}
\frac{1}{2} \eta_{D}=\operatorname{index}(\not D)-\int_{B} A(\hat{R}), \\
\frac{1}{2} \eta_{R}=\operatorname{index}(R)-\operatorname{index}(\not D)+\int_{B}(K(R)-\hat{A}(R)), \\
\eta_{S}=\sigma-\int_{B} L(R),
\end{gathered}
$$

where index $(\not D)$ and index $(R)$ are the Dirac and Rarita-Schwinger index on $B$ and $\sigma$ is the signature of $B ; \hat{A}, K$, and $L$ are the polynomials in the curvature tensor $R$ whose integral over a closed twelve manifold $B$ would give index $(\not D)$, index $(R)$, and $\sigma$. The second equation in (47) involves the combinations (index $(R)$-index $(\not D)$ ) and $\left(K(R)-(\hat{A}(R))\right.$ for the same reason that $\left(\eta_{R}-\eta_{D}\right)$ appears in (44); a vector field on $B$ restricts to a vector plus scalar on $\partial B=\left(M \times S^{1}\right)_{\pi}$.

Combining (46) and (47), the change in the action under a diffeomorphism is

$$
\begin{aligned}
\Delta I= & 2 \pi i\left(\frac{1}{2} N_{D} \text { index }(\not D)+\frac{1}{2} N_{R}(\operatorname{index}(R)-2 \operatorname{index}(\not D))-\frac{1}{8} N_{S} \sigma\right) \\
& -2 \pi i \int_{B}\left(\left(\frac{N_{D}}{2}\right) \hat{A}(R)+\frac{N_{R}}{2}(K(R)-2 \hat{A}(R))-\frac{1}{8} N_{S} L(R)\right) \bmod 2 \pi i .
\end{aligned}
$$

It may still not be immediately apparent that $\Delta I$ depends on $M$ and $\pi$ only. However, precisely in a theory that is free of perturbative anomalies, the curvature integral in (48) cancels, because the combination of characteristic classes that enters is precisely the one that enters in evaluating perturbative anomalies on $M$. Thus, consider in 10 dimensions the anomaly free $N=2$ supergravity theory with $N_{D}=-2, N_{R}=2, N_{S}=1$. The curvature integrals cancel, and the index $(\not D)$ and 
index $(R)$ terms can be dropped $\bmod 2 \pi i$. Hence (47) reduces to

$$
\Delta I=-2 \pi i \frac{\sigma(B)}{8} \bmod 2 \pi i
$$

which is our final result for this theory.

$\Delta I$ is a topological invariant, because we have seen in Sect. II that $\bmod 16, \sigma(B)$ depends only on the topology of $\partial B=\left(M \times S^{1}\right)_{\pi}$. It should be recalled that (49) is only to be believed if (for $M$ of 10 dimensions) $b_{5}(M)=0$; and even so, the derivation involved some highly plausible but unproved assumptions about antisymmetric tensor fields.

We can now readily see that the $N=2$ theory has no anomalies if formulated on $S^{10}$. As explained in Sect. II, every exotic eleven sphere $S_{\pi}^{11}$, and every space $\left(S^{10}\right.$ $\left.\times S^{1}\right)_{\pi} \cong\left(S^{10} \times S^{1}\right)+S_{\pi}^{11}$, bounds a manifold $B$ of signature divisible by 8 . Hence $\Delta I=0 \bmod 2 \pi i$.

To discuss reduction to four dimensions, one must evaluate (49) with $M=S^{4} \times K$ for various $K$; we will not explore this here.

\section{The $O(32)$ and $E_{8} \times E_{8}$ Theories}

With relief we now leave the mathematical complexities of the antisymmetric tensor field, and turn to the phenomenologically more promising cases of $\mathrm{O}(32)$ and $E_{8} \times E_{8}$. We temporarily ignore the gauge fields and consider only the gravitational contributions.

In these theories, the only fields with anomalous determinant are the Dirac and Rarita-Schwinger fields, with $N_{D}=495, N_{R}=1$. From our results in the last section, we can immediately write down the change in determinant under a diffeomorphism $\pi$ of some ten manifold $M$ :

$$
\Delta I_{\mathrm{det}}=\pi i\left(\frac{494}{2} \eta_{D}+\frac{1}{2} \eta_{R}\right)=\pi i\left(494 \operatorname{index}_{B}(\not D)+\operatorname{index}_{B}(R)\right)-\pi i \int_{B}(493 \hat{A}(R)+K(R)) .
$$

Here $B$ is a spin manifold whose boundary is $\left(M \times S^{1}\right)_{\pi}$; index $_{B}(\not D)$ and index $(R)$ are the Dirac and Rarita-Schwinger index on $B$; and we have used the AtiyahPatodi-Singer theorem as in Sect. IV. Now, we saw in Sect. II that in twelve dimensions index ${ }_{B}(\not D)$ and index $x_{B}(R)$ are always even, so - since we only want $\Delta I$ modulo $2 \pi i$ - we may drop these terms in (50). However, we must discuss the curvature integral in (50), which is not a topological invariant since $B$ has a boundary. This curvature integral - the only surviving term in (50) - is

$$
\Delta I_{\mathrm{det}}=\frac{-i}{(2 \pi)^{6}} \int_{B}\left(\frac{1}{1536}\left(\operatorname{Tr} R^{2}\right)^{3}+\frac{1}{384}\left(\operatorname{Tr} R^{2}\right)\left(\operatorname{Tr} R^{4}\right)\right)
$$

Now, $\int_{B} \operatorname{Tr} R^{6}$ has no generalization which is a topological invariant, as we discussed in Sect. II. Luckily, for entirely different reasons the analysis of Green and Schwarz singles out the case $N_{D}=495, N_{R}=1$ for which there is no $\operatorname{Tr} R^{6}$ term. As for the terms $\int_{B}\left(\operatorname{Tr} R^{2}\right)^{3}, \int_{B}\left(\operatorname{Tr} R^{2}\right) \cdot\left(\operatorname{Tr} R^{4}\right)$, they have generalizations which are 
topological invariants if on $\left(M \times S^{1}\right)_{\pi}$ we can solve the equation $\operatorname{Tr} R^{2}=d H, \operatorname{Tr} R^{4}$ $=d K$. Now, happily the theories in question only make sense if the equation $\operatorname{Tr} R^{2}$ $=d H$ can be solved on $M$, since the theories contain a physical field $H$ which is supposed to obey this equation. Also, with four or more uncompactified dimensions (the relevant cases, presumably!), we always have $\operatorname{Tr} R^{4}=d K$ on $M$. Now, we would be unable to solve the equations $\operatorname{Tr} R^{2}=d H, \operatorname{Tr} R^{4}=d K$ on $\left(M \times S^{1}\right)_{\pi}$ if these had no solutions when restricted to $M$, but the existence of solutions on $M$ does not guarantee the existence of solutions on $\left(M \times S^{1}\right)_{\pi}$. In this section, we consider cases in which $H$ and $K$ can be extended to $\left(M \times S^{1}\right)_{\pi}$ (this is always so if $M$ is $\left.S^{10}\right)$. An interesting phenomenon arises when the extension of $H$ and $K$ to $\left(M \times S^{1}\right)_{\pi}$ does not exist; it will be our subject in Sect. VI.

Given that $H$ and $K$ exist on $\left(M \times S^{1}\right)_{\pi}$, Eq. (50) has a generalization which is a topological invariant and is obtained by adding

$$
\frac{i}{(2 \pi)^{6}} \underset{\left(M \times S^{1}\right)_{\pi}}{ } H\left(\frac{1}{1536}\left(\operatorname{Tr} R^{2}\right)^{2}+\frac{1}{384} \operatorname{Tr} R^{4}\right) .
$$

How can such a contribution arise? There are two possible sources. Apart from matter determinants, we must consider the variation of the anomalous terms in the action that were added by Green and Schwarz. As for the determinants, Eq. (50) includes contributions of the massless fields [the $1 / \lambda_{j}$ in Eq. (20)] but not of the regulators [the $1 /\left(\lambda_{j}+i M\right)$ in that equation]. We will have to include the regulator terms.

We first consider the anomalous terms in the action. The purely gravitational terms introduced by Green and Schwarz were

$$
\tilde{S}=\frac{i}{(2 \pi)^{6}} \int\left(\frac{1}{1536} B\left(\operatorname{Tr} R^{2}\right)^{2}+\frac{1}{384} B \operatorname{Tr} R^{4}-\frac{1}{576} \omega_{3}^{L} \omega_{7}^{L}\right) .
$$

Here $B$ is the antisymmetric second rank tensor of the supergravity multiplet, and $\omega_{3}^{L}$ and $\omega_{7}^{L}$ are the Lorentz Chern-Simons three and seven forms. The gauge invariant field strength of $B$ is $H=d B+\omega_{3}^{L}$ (recall that we are temporarily ignoring gauge fields).

Now, recall that we represented $\left(M \times S^{1}\right)_{\pi}$ with a metric $d t^{2}+g_{\mu \nu}^{t} d x^{\mu} d x^{\nu}, 0 \leqq t$ $\leqq 1 ; t=1$ and $t=0$ are identified via $\pi$. The change in $S$ is

$$
\begin{aligned}
\Delta \tilde{S}= & \tilde{S}(1)-\tilde{S}(0)=\left[\frac{i}{(2 \pi)^{6}} \int_{M}\left(\frac{1}{1536} B\left(\operatorname{Tr} R^{2}\right)^{2}+\frac{1}{384} B \operatorname{Tr} R^{4}-\frac{1}{576} \omega_{3}^{L} \omega_{7}^{L}\right)\right]_{t=0}^{t=1} \\
= & \frac{i}{(2 \pi)^{6}} \int_{0}^{1} d t \int_{M} \frac{d}{d t}\left(\frac{1}{1536} B\left(\operatorname{Tr} R^{2}\right)^{2}+\frac{1}{384} B \operatorname{Tr} R^{4}+\frac{1}{576} \omega_{3}^{L} \omega_{7}^{L}\right) \\
= & \frac{i}{(2 \pi)^{6}} \int_{0}^{1} d t \int_{M}\left(\frac{1}{1536}(d B)\left(\operatorname{Tr} R^{2}\right)^{2}+\frac{1}{384}(d B) \operatorname{Tr} R^{4}\right. \\
& -\frac{1}{576} d\left(\omega_{3}^{L}\left(\omega_{7}^{L}\right)\right) .
\end{aligned}
$$

(We have used Stokes' theorem and the fact $d \operatorname{Tr} R^{2}=d \operatorname{Tr} R^{4}=0$.) This is clearly on the right track; if we can replace $d B$ by its covariant generalization $H=d B+\omega_{3}^{L}$, 
we see in $\Delta \tilde{S}$ the sort of terms needed to make a topological invariant out of Eq. (51). The extra pieces must come from the regulator pieces in (20), so we must study

$$
(\Delta I)_{\operatorname{Reg}}=-\frac{1}{4} \sum_{Q} \int_{0}^{1} d t \sum_{j} \frac{1}{\lambda_{j}+i M}\left\langle\psi_{j}\left|\frac{d}{d t}(i \not D) \bar{\Gamma}\right| \psi_{j}\right\rangle,
$$

where $\sum_{Q}$ represents a sum over multiplets, $\sum_{j}$ represents a sum over eigenstates and eigenvalues $\psi_{j}$ and $\lambda_{j}$, and $\bar{\Gamma}$ is the chirality operator. Evaluation of (54) is closely related to evaluation of anomalies in one loop determinants; the connection was explained in detail in [32]. It is clear that because of the large regulator mass, (54) equals the integral over $\left(M \times S^{1}\right)_{\pi}$, of a local function of the spin connection and its derivatives. It is clear, as well, that this must be a pseudoscalar function because of the factor of $\bar{\Gamma}$. It can be shown [32], in fact, that one obtains precisely a linear combination of Chern-Simons terms [with precisely the combination of invariants that is related to perturbative gravitational anomalies; this is why $(\Delta I)_{\text {Reg }}$ cancelled out and did not have to be considered in Sect. IV]. By the methods of [32] it can be shown that one gets in this case

$$
\begin{aligned}
\Delta I_{\mathrm{Reg}} & =\frac{1}{(2 \pi)^{6}} \int_{0}^{1} d t \int_{M}\left[\frac{1}{1536} \omega_{3}^{L}\left(\operatorname{Tr} R^{2}\right)^{2}+\frac{1}{1152} \omega_{3}^{L} \operatorname{Tr} R^{4}+\frac{1}{576} \operatorname{Tr} R^{2} \omega_{3}^{L}\right] \\
& =\frac{1}{(2 \pi)^{6}} \int_{0}^{1} d t \int_{M}\left(\frac{1}{1536} \omega_{3}^{L}\left(\operatorname{Tr} R^{2}\right)^{2}+\frac{1}{384} \omega_{3}^{L} \operatorname{Tr} R^{4}+\frac{1}{576} d\left(\omega_{3}^{L} \omega_{7}^{L}\right)\right) .
\end{aligned}
$$

(The first form given in Eq. (55) comes directly, as in [32], from the formal 12-form expression for the anomaly; the second comes by integrating by parts.) Adding the two, we get

$$
\Delta \widetilde{S}+\Delta I_{\mathrm{Reg}}=\frac{i}{(2 \pi)^{6} \cdot 48} \underset{\left(M \times S^{1}\right)_{\pi}}{\int} H \cdot\left[\frac{1}{32}\left(\operatorname{Tr} R^{2}\right)^{2}+\frac{1}{8} \operatorname{Tr} R^{4}\right],
$$

where $H=d B+\omega_{3}^{L}$. Thus, the non-covariant objects $d B$ and $\omega$ combine, as they must, into the covariant field strength $H .\left(\Delta \tilde{S}\right.$ and $\Delta I_{\text {Reg }}$ were invariant under coordinate transformations that vanish at $t=0$ and $t=1$, but not otherwise. As far as I know, it does not make sense to identify $t=1$ with $t=0$ by means of a nontrivial diffeomorphism $\pi$ until these expressions are combined into a covariant form.) Thus we finally get an expression for the change in the action in terms of topological invariants:

$$
\begin{aligned}
\Delta I_{\mathrm{TOT}}= & \Delta I_{\mathrm{det}}+\Delta \tilde{S}+\Delta I_{\mathrm{Reg}} \\
= & -2 \pi i\left[\frac{1}{(2 \pi)^{6}} \int_{B}\left(\frac{1}{1536}\left(\operatorname{Tr} R^{2}\right)^{3}+\frac{1}{384} \operatorname{Tr} R^{2} \operatorname{Tr} R^{4}\right)\right. \\
& \left.-\int_{\left(M \times S^{1}\right) \pi} H \cdot\left(\frac{1}{1536}\left(\operatorname{Tr} R^{2}\right)^{2}+\frac{1}{384} \operatorname{Tr} R^{4}\right)\right] \\
= & -2 \pi i \cdot \frac{1}{192}\left[-3 p_{1}^{3}(B)+4 p_{1} p_{2}(B)\right],
\end{aligned}
$$

where $p_{1}^{3}$ and $p_{1} p_{2}$ were defined in Sect. II.

Let us pause a bit to make sure that the right-hand side of (57) is really a property only of $\left(M \times S^{1}\right)_{\pi}$, and does not depend on the choice of $B$. The argument 
is similar to previous ones. Let $X$ and $X^{\prime}$ be two different spin manifolds with boundary $\left(M \times S^{1}\right)_{\pi}$. The two choices lead to two formulas $\Delta I^{X}$ and $\Delta I^{X^{\prime}}$ for the change in the action:

$$
\begin{aligned}
\Delta I^{X}= & -2 \pi i\left[\int_{X}\left(\frac{1}{1536}\left(\operatorname{Tr} R^{2}\right)^{3}+\frac{1}{384} \operatorname{Tr} R^{2} \operatorname{Tr} R^{4}\right)\right. \\
& \left.-\int_{\left(M \times S^{1}\right)_{\pi}} H \cdot\left(\frac{1}{1536}\left(\operatorname{Tr} R^{2}\right)^{2}+\frac{1}{384} \operatorname{Tr} R^{4}\right)\right], \\
\Delta I^{X^{\prime}}= & -2 \pi i\left[\int_{X^{\prime}}\left(\frac{1}{1536}\left(\operatorname{Tr} R^{2}\right)^{3}+\frac{1}{384} \operatorname{Tr} R^{2} \operatorname{Tr} R^{4}\right)\right. \\
& \left.-\int_{\left(M \times S^{1}\right)_{\pi}} H \cdot\left(\frac{1}{1536}\left(\operatorname{Tr} R^{2}\right)^{2}+\frac{1}{384} \operatorname{Tr} R^{4}\right)\right] .
\end{aligned}
$$

In the difference, the boundary integral cancels out, and one gets an integral over the closed manifold $\bar{X}=X+\left(-X^{\prime}\right)$ :

$$
\Delta I^{X}-\Delta I^{X^{\prime}}=-2 \pi \mathrm{i} \int_{\bar{X}}\left(\frac{1}{1536}\left(\operatorname{Tr} R^{2}\right)^{3}+\frac{1}{384} \operatorname{Tr} R^{2} \operatorname{Tr} R^{4}\right) .
$$

But this can be expressed in terms of the Dirac and Rarita-Schwinger index on $\bar{X}$ :

$$
\Delta I^{\bar{X}}-\Delta I^{\bar{X}^{\prime}}=-\pi i\left(493 \operatorname{index}_{\bar{X}}(\mathrm{D})+\operatorname{Index}_{\bar{X}}(\text { R.S. })\right)=0 \bmod 2 \pi i,
$$

since the Dirac and Rarita-Schwinger indexes are even in twelve dimensions.

For an eleven dimensional spin manifold $M$, let

$$
\mu(M)=\frac{1}{192}\left[-3 p_{1}^{3}+4 p_{1} p_{2}\right] \bmod 1 .
$$

As in the discussion of $\lambda$ in Sect. II, $\mu$ is additive in connected sums. This is useful for calculating it. For instance, using facts described in Sect. II, we can now settle the important question of whether the $\mathrm{O}(32)$ and $E_{8} \times E_{8}$ theories have global anomalies when formulated on $S^{10}$. In fact, $\left(S^{10} \times S^{1}\right)_{\pi}$ is the connected sum of $S_{\pi}^{11}$ and $S^{1} \times S^{10}$, so $\mu\left(\left(S^{10} \times S^{1}\right)_{\pi}\right)=\mu\left(S_{\pi}^{11}\right)+\mu\left(S^{1} \times S^{10}\right)$. But $\mu\left(S_{\pi}^{11}\right)=0$, since any exotic eleven sphere bounds a parallelizable manifold of $R=p_{1}=p_{2}=0$ (that is, $R=0$ for some connection with torsion, and $\left.p_{1}=p_{2}=0\right)$, and $\mu\left(S^{1} \times S^{10}\right)=0$, since $S^{1} \times S^{10}$ bounds $D^{2} \times S^{10}\left(D^{2}\right.$ is a two dimensional disc $)$, and $D^{2} \times S^{10}$ has $p_{1}^{3}=p_{1} p_{2}$ $=0$ (since, for instance, the standard metric on $D^{2} \times S^{10}$ has $\operatorname{Tr} R^{2}=\operatorname{Tr} R^{4}=0$ everywhere).

It is noteworthy that $\mu$ is quite reminiscent of the invariant $\lambda$ that characterizes exotic spheres. But $\mu$ fails to detect the strangeness of exotic spheres because the signature term is absent in (61).

It is not difficult to generalize these considerations to gauge anomalies. Here we begin with a ten manifold $M$ on which an $\mathrm{O}(32)$ or $E_{8} \times E_{8}$ bundle $V$ is given. For the theory to be consistent [31], $M$ and $V$ must be chosen so that $\operatorname{Tr} R^{2}-\frac{1}{30} \operatorname{Tr} F^{2}$ $=d H$ for some globally defined three form $H$ ( $F$ is the curvature in the adjoint representation of $\mathrm{O}(32)$ or $\left.E_{8} \times E_{8}\right)$. Now we consider a gauge and/or coordinate transformation $\pi$, via which we build a manifold $\left(M \times S^{1}\right)_{\pi}$ with bundle $V_{\pi}$. Assuming $H$ can be extended to $\left(M \times S^{1}\right)_{\pi}$, the modifications in the above derivation are relatively trivial. [The variation of the Dirac determinant, for

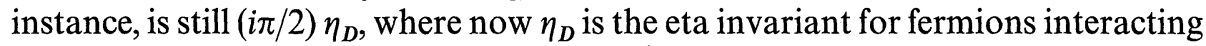
with the metric and gauge field on $\left(M \times S^{1}\right)_{\pi}$.] The change in the action can be 
expressed in a simple way in terms of a twelve dimensional spin manifold $X$ which is bounded by $\left(M \times S^{1}\right)_{\pi}$ and to which the bundle $V_{\pi}$ can be extended. It is

$$
\Delta I=-2 \pi i \mu\left(\left(M \times S^{1}\right)_{\pi}\right)
$$

where

$$
\begin{aligned}
\mu\left(\left(M \times S^{1}\right)_{\pi}\right)= & \frac{1}{(2 \pi)^{6}} \frac{1}{384} \int_{B}\left(\operatorname{tr} R^{2}-\frac{1}{30} \operatorname{Tr} F^{2}\right)\left[\frac{1}{4}\left(\operatorname{tr} R^{2}\right)^{2}+\operatorname{tr} R^{4}\right. \\
& \left.-\frac{1}{30} \operatorname{tr} R^{2} \operatorname{Tr} F^{2}-\frac{1}{900}\left(\operatorname{Tr} F^{2}\right)^{2}+\frac{1}{3} \operatorname{Tr} F^{4}\right] \\
& -\frac{1}{(2 \pi)^{6}} \frac{1}{384} \int_{\left(M \times S^{1}\right)_{\pi}} H\left(\frac{1}{4}\left(\operatorname{tr} R^{2}\right)^{2}+\operatorname{tr} R^{4}\right. \\
& \left.-\frac{1}{30} \operatorname{tr} R^{2} \operatorname{Tr} F^{2}-\frac{1}{900}\left(\operatorname{Tr} F^{2}\right)^{2}+\frac{1}{3} \operatorname{Tr} F^{4}\right) .
\end{aligned}
$$

This can, of course, be reexpressed in terms of Pontryagin classes. The condition for invariance of the theory under $\pi$ is that $\mu=0 \bmod 1$. In the future, this formula may have many applications in restricting compactification to four dimensions. A special case is the subject of the next section.

\section{Dirac-Like Condition for Antisymmetric Tensor Fields}

Since antisymmetric tensor fields were first intorudced [33], their analogy with Abelian gauge fields has been striking, and it has been natural to ask whether they might obey an analogue of a Dirac quantization condition [35]. Thus consider a second rank tensor field $B$, with gauge invariance $B \rightarrow B+d \lambda$, and suppose that the gauge invariant field strength $H$ is $H=d B$, so $d H=0$ [this is not quite so in the $\mathrm{O}(32)$ and $E_{8} \times E_{8}$ models, which we will come to shortly]. Let $S$ be a closed three surface in the (compact, Euclidean) space-time manifold $M$, and let

$$
Q(S)=\int_{S} d \Sigma^{i j k} H_{i j k} \equiv \int_{S} H
$$

Then $Q(S)$ depends only on the topological class of $S$; if $S$ can be deformed into $S^{\prime}$, then $Q(S)-Q\left(S^{\prime}\right)=\int_{V} d H=0$, where $V$ is a four surface in $M$ whose boundary is $S-S^{\prime}$. A Dirac-like condition would be that for all $S, Q(S)=n q_{0}$, where $n$ is an integer depending on the topological class of $S$ and $q_{0}$ is a basic flux quantum of some kind.

There were always many difficulties with this idea. Antisymmetric tensor fields never appeared in the sort of non-linear structures that lead to quantization conditions. It never was clear how to determine $q_{0}$.

The recent developments $[34,5]$ cast a new light on this subject, for several reasons. On the one hand, it is no longer so that $d H=0$; rather, $d H=\operatorname{tr} R^{2}$ $-1 / 30 \operatorname{Tr} F^{2}$ [with $F$ in the adjoint representation of $\mathrm{O}(32)$ or $E_{8} \times E_{8}$ ]. Hence for neighboring surfaces $S$ and $S^{\prime}, Q(S)-Q\left(S^{\prime}\right)=\int_{V} d H=\int_{V}\left(\operatorname{tr} R^{2}-1 / 30 \cdot \operatorname{Tr} F^{2}\right)$, which is non-zero in general. So $Q(S)$ can vary continuously as $S$ is varied in space-time. This seems to disprove the idea of quantization. How can we quantize something whose value depends continuously on the choice of $S$ ? 
Nevertheless, the $\mathrm{O}(32)$ theory does lead to a sort of quantization condition on $H$. Although the argument given here does not generalize to $E_{8} \times E_{8}$, a similar quantization law holds in that case because of multivaluedness of the WessZumino interaction. This will be discussed in detail elsewhere [36]. Depending on $M$, it is rather difficult to formulate and prove the precise conditions. We will therefore illustrate the idea with a simple example of compactification to seven dimensional Minkowski space times $S^{3}$. (Further compactification to four dimensions would not greatly change the argument.) The Euclidean form of this is $M=S^{7} \times S^{3}$. We consider first a state of $F_{\mu \nu}=0$, and the standard metric on $S^{7}$ $\times S^{3}$.

Now, $\pi_{7}(\mathrm{O}(32)) \cong Z$. (It is because $\pi_{7}\left(E_{8}\right)=0$ that our considerations have no obvious generalization to $E_{8} \times E_{8}$.) Let $\pi$ be the generator of $\pi_{7}(\mathrm{O}(32))$. We wish to ask whether the $\mathrm{O}(32)$ theory formulated on $S^{7} \times S^{3}$ is invariant under $\pi$. As usual, the basic tool is to study $W=\left(S^{7} \times S^{3} \times S^{1}\right)_{\pi}$. In this case, $\pi$ acts trivially on $S^{7} \times S^{3}$, so $\left(S^{7} \times S^{3} \times S^{1}\right)_{\pi}$ is simply the product $S^{7} \times S^{3} \times S^{1}$ with a non-trivial O(32) instanton field $A$ on it. This field has the property that

$$
\frac{1}{(2 \pi)^{4} \cdot 172,800} \int_{S^{7} \times S^{1}} \operatorname{Tr} F^{4}=1, \quad \int_{S^{7} \times S^{1}}\left(\operatorname{Tr} F^{2}\right)^{2}=0 .
$$

To use our basic formula that the action is invariant if $\mu=0$ [Eq. (63)], we must find a spin manifold bounded by $W$ to which the $\mathrm{O}(32)$ gauge field on $W$ can be extended. Here we can take this to be $X=D^{4} \times\left(S^{7} \times S^{1}\right)_{\pi}$, where $D^{4}$ is a four dimensional ball bounded by $S^{3}$. In this case, with the standard metric on $X, \operatorname{Tr} R^{2}$ $=\operatorname{Tr} R^{4}=0$ pointwise, and all terms involving $R$ may be dropped in the definition of $\mu$. We get

$$
\begin{aligned}
\mu= & \frac{1}{(2 \pi)^{6}} \frac{1}{1,152} \int_{B}\left(\frac{\operatorname{Tr} F^{2}}{30}\right)\left(\frac{1}{300}\left(\operatorname{Tr} F^{2}\right)^{2}-\operatorname{Tr} F^{4}\right) \\
& -\frac{1}{(2 \pi)^{6}} \frac{1}{1,152} \underset{\left(M \times S^{1}\right)_{\pi}}{\int} H\left(\frac{1}{300}\left(\operatorname{Tr} F^{2}\right)^{2}-\operatorname{Tr} F^{4}\right) .
\end{aligned}
$$

To evaluate this formula, we must first of all discuss $H$. On $M=S^{7} \times S^{3}$, we may take $H=0$, or more generally any $H$ such that $d H=0$, since $\operatorname{Tr} R^{2}=\operatorname{Tr} F^{2}=0$ for the standard metric on $S^{7} \times S^{3}$ with $F=0$. To obey $d H=0$ it is sufficient but not necessary to take $H=d P$ for some $P$. Let $\alpha$ be a multiple of the Levi-Civita tensor of $S^{3}$, normalized so $\int_{S^{3}} \alpha=1$. The general solution of $d H=0$ is $H=\mathrm{dK}+\mathrm{t} \alpha$ with some real number $t$.

$H$ is a physical field like any other, so the value of $H$ on $M$ is part of the data of the physics problem under discussion. Equation (65) presumes that $H$ can be extended to $\left(M \times S^{1}\right)_{\pi}$, and this is indeed possible in the case at hand. There is no particularly natural way to extend $H$ to $\left(M \times S^{1}\right)_{\pi}$, and $\mu$ must be independent of the choice of extension, but $\mu$ can depend on the value of $H$ on $M$ as on any other physical data.

In Sect. II, we discussed the condition for formulas such as (65) to be independent of the choice of solution $H$ of $d H=\operatorname{Tr} F^{2}$. (The discussion was carried out for gravitation, but generalizes immediately to gauge theories.) The require- 
ment is that $\operatorname{Tr} F^{4}=d K$ on $\left(M \times S^{1}\right)_{\pi}$. In the case at hand, $\operatorname{Tr} F^{4}=0$ (pointwise) on $M$, so this equation is certainly soluble on $M$. But it cannot be solved on $\left(M \times S^{1}\right)_{\pi}$. Indeed, the equation $\operatorname{Tr} F^{4}=d K$ cannot be solved on the subspace $\left(S^{7} \times S^{1}\right)_{\pi}$ of $\left(M \times S^{1}\right)_{\pi}$, much less on all of $\left(M \times S^{1}\right)_{\pi}$, because $\int_{\left(S^{7} \times S^{1}\right)_{\pi}} \operatorname{Tr} F^{4} \neq 0$. Because of this, the value of $\mu$ will depend on $H$ [but only on the value of $H$ on $M$, not its extension to $\left(M \times S^{1}\right)_{\pi}$. Therefore, the equation for invariance of the theory under $\pi, \mu=0$, imposes a condition on $H$, which will turn out to be a quantization condition.

It is, in fact, very easy to evaluate $\mu$ and find the restriction on $H$. The gauge field on $S^{3} \times\left(S^{7} \times S^{1}\right)_{\pi}$ was the product of the zero gauge field on $S^{3}$ and an instanton on $\left(S^{7} \times S^{1}\right)_{\pi}$. It can be extended to $X$ very simply as the product of the zero gauge field on $D^{4}$ with an instanton on $\left(S^{7} \times S^{1}\right)_{\pi}$. With thic choice, all terms in $\mu$ disappear except one:

$$
\mu=\frac{1}{(2 \pi)^{6} \cdot 1,152} \int_{\left(S^{7} \times S^{1}\right)_{\pi}} \operatorname{Tr} F^{4} \cdot \int_{S^{3}} H=\frac{150}{(2 \pi)^{2}} \int_{S^{3}} H(\bmod 1) .
$$

Hence, the condition on $H$ is

$$
\int_{S^{3}} H=\frac{\left(2 \pi^{2}\right)}{150} \cdot n
$$

where $n$ may be any integer. This condition indeed depends on the value of $H$ on $M$ only.

This problem was unrepresentative because we took data on $M$ such that $\operatorname{Tr} R^{2}$ $=\operatorname{Tr} F^{2}=0$. As a result, we did not directly face the problem that $\int_{S^{3}} H$ changes continuously when $S^{3}$ is varied. A more typical situation is to consider on $S^{7} \times S^{3}$ a generic metric and gauge field with curvature and field strength $R$ and $F$. One then finds that vanishing of $\mu$ still gives a condition on $H$, but this condition is harder to express. One way to express the result is that if $H_{0}$ is one $H$ field for which $\mu=0$, the general one is $H=H_{0}+H_{1}$, where $H_{1}$ must obey $d H_{1}=0, \int_{S^{3}} H_{1}=\frac{(2 \pi)^{2}}{150} \cdot n$. Another way to express the answer is that if $S^{3 *}$ is some particular copy of $S^{3}$ in $S^{7}$ $\times S^{3}$, then an allowed $H$ must obey $\int_{S^{3 *}} H=\frac{(2 \pi)^{2}}{150}(n+\delta)$, where the "deficit" $\delta$ is independent of $n$ but depends on the metric and gauge field of $M$ and the choice of $S^{3 *}$. The derivation of these statements is similar to the derivation we gave for the special case of the standard metric and zero gauge field on $S^{7} \times S^{3}$.

Spaces such as $M=S^{4} \times S^{3} \times S^{3}$ (where $S^{4}$ can be regarded as the compactification of four dimensional Minkowski space) can be treated similarly (one gets restrictions on the integral of $H$ over either $S^{3}$ ). For $M=S^{4} \times S^{1} \times S^{1} \times S^{1} \times S^{1}$ $\times S^{1} \times S^{1}$, one gets twenty restrictions (one for the integral of $H$ over any product of three $S^{1}$ 's). If $M=S^{4} \times K$ with a more complicated $K$, no restrictions will arise if there are no non-trivial three surfaces in $K$ so that $H^{3}(K)=0$. If $H^{3}(K) \neq 0$, then $H^{7}(M) \neq 0$. The number of quantization conditions will equal the rank of the subspace of $H^{7}(M)$ that can be realized as the pullback to $M$ of elements of 
$H^{7}(\mathrm{O}(32))$ via mappings $\phi: M \rightarrow \mathrm{O}(32)$. It might be that in general the number of quantization conditions is less than the number of independent three surfaces.

\section{Conclusion}

In conclusion, I would like to consider a question which may have puzzled the reader. If the $\mathrm{O}(32)$ or $E_{8} \times E_{8}$ theories had turned out to have global anomalies when formulated on $S^{10}$, would this have been good or bad?

If one of these theories is correct, we must sooner or later find an explanation for the compactification which must occur. Could it have been that one of these theories would have a global anomaly on $S^{10}$ but not on some other space $M=S^{4}$ $\times K$ ? Any orientation preserving diffeomorphism $\pi$ of $S^{10}$ always has an analogue in the diffeomorphism group of any ten manifold $M$. In fact, $\pi$ (or rather, an element of its topological class) can always be chosen to vanish outside a ten dimensional ball $B$ embedded in $S^{10}$. B can be embedded in any $M$, and by letting $\pi$ act on this embedded copy of $B$, we get a diffeomorphism of $M$ that we will again call $\pi$.

Now, $\left(M \times S^{1}\right)_{\pi} \cong\left(M \times S^{1}\right)+S_{\pi}^{11}$. The proof of this was summarized in Fig. 4 (for the special case $M=S^{10}$, but this argument generalizes). Since the invariant $\mu$ relevant for global anomalies is additive in connected sums, and since $\mu\left(M \times S^{1}\right)=0$ for any $M$, we get $\mu\left(M \times S^{1}\right)_{\pi}=\mu\left(S_{\pi}^{11}\right)$ for any $M$. Hence if there were global anomalies on $S^{10}$, so $\mu\left(S_{\pi}^{11}\right) \neq 0$ for some $\pi$, the theory would be inconsistent on any $M$; it would be nonsense.

Thus, global anomalies could not have been expected to explain compactification. Actually, rather than eliminating global anomalies, compactification might generate them, since $M=S^{4} \times K$ might have diffeomorphism (or gauge transformation) classes that do not come from any diffeomorphism (or gauge transformation) class of $S^{10}$. We discussed an example of this in Sect. VI.

It is not arbitrary diffeomorphisms of $S^{4} \times K$ that should be investigated. Let $p \in S^{4}$ be "the point at infinity," an arbitrary point. We want diffeomorphisms of $R^{4} \times K$ that vanish at infinity. (Here $R^{4}$ is four dimensional Euclidean space, which will become four dimensional Minkowski space after Wick rotation.) Physically, the study of global anomalies should be concerned with diffeomorphisms of $R^{4} \times K$ that vanish at infinity, and hence with diffeomorphisms that leave fixed a neighbourhood of $\{p\} \times K$.

The choice of $S^{4}$ corresponds to treating four dimensional space time as Minkowski space. In the long run, a more delicate choice will be necessary to accommodate cosmological considerations. It may be that eventually global anomalies will have cosmological applications, restricting the large scale topology of space-time.

\section{References}

1. Adler, S.: Axial-vector vertex in spinor electrodynamics. Phys. Rev. 177, 2426 (1969) Bell, J., Jackiw, R.: A PCAC puzzle: $\pi^{0} \rightarrow \gamma \gamma$ in the $\sigma$-model. Nuovo Cimento 60 A, 47 (1969)

2. Gross, D.J., Jackiw, R.: Effect of anomalies on quasi-renormalizable-theories. Phys. Rev. D 6, 477 (1972); 
Bouchiat, C., Iliopoulos, J., Meyer, Ph.: An anomaly-free version of Weinberg's model. Phys. Lett. 38 B, 519 (1972);

Georgi, H., Glashow, S.: Gauge theories without anomalies. Phys. Rev. D 6, 429 (1972)

3. Frampton, P.H., Kephart, T.W.: Explicit evaluation of anomalies in higherdimensions and Consistency conditions for Kaluza-Klein anomalies. Phys. Rev. Lett. 50, 1343, 1347 (1983); Townsend, P.K., Sierra, G.: (LPTENS preprint, 1983)

Zumino, B., Yong-shi, W., Zee, A.: Nucl. Phys. B 239, 477 (1984); Matsuki, Y., Hill, A.: (OSU preprint, 1983)

4. Alvarez-Gaumé, L., Witten, E.: Gravitational anomalies. Nucl. Phys. B 234, 269 (1983)

5. Green, M.G., Schwarz, J.H.: Anomaly cancellations in supersymmetric $D=10$ gauge theory and superstring theory. Phys. Lett. 149 B, 117 (1984)

6. Schwarz, J.H.: Superstring theory. Phys. Rep. 89, 223 (1982);

Green, M.B.: Supersymmetrical dual string theories and their field theory limits - a review. Surv. High Energ. Phys. 3, 127 (1983)

7. Gross, D.J., Harvey, J., Martinec, E., Rohm, R.: Princeton preprint (1984). The suggestion that rank 16 is the difference between 26 dimensions of the Veneziano model and ten of the superstring was made by P.G.O. Freund (private communication and Santa Barbara preprint (1984)

See also J. Thierry-Mieg, Berkeley preprint (1984)

8. Deser, S., Jackiw, R., Templeton, S.: Three-dimensional massive gauge theories. Phys. Rev. Lett. 48, 975 (1982)

9. Witten, E.: An SU(2)anomaly. Phys. Lett. 117 B, 324 (1982)

10. Elitzur, S., Nair, V.P.: IAS preprint (1984)

11. Witten, E.: Global aspects of current algebra. Nucl. Phys. B 223, 422 (1983)

12. Harvey, J.: Unpublished

13. Smale, S.: Ann. Math., II. Ser. 74, 392 (1961); Proc. Am. Math. Soc. 10, 621 (1950)

14. Milnor, J.: Ann. Math., II. Ser. 643991 (1956)

15. Cerf, J.: La stratification naturelle, ... et la theoreme de la pseudo-isotope, I.H.E.S. Publ. Math. 39, 5-173

16. Milnor, J.: In Differential and combinatorial topology, Princeton, NJ: Princeton University Press 1965

17. Eells, J., Kuiper, N.: Ann. Math. Pure Appl. 60, 63 (1963)

18. Milnor, J.: In Lectures on modern mathematics, pp. 165-183, Saaty, T.L., ed. New York: Wiley 1963

19. Kervaire, M.A., Milnor, J.W.: Ann. Math. 77, 504 (1963)

20. Wetterich, C.: Massless spinors in more than four dimensions. Nucl. Phys. B 211, 177 (1982)

21. Atiyah, M.F., Singer, I.M.: Ann. Math. 93, 1, 119, 139 (1971)

22. Ochanine, S.: Math. Notes 13, 57 (1973), Math. Reviews 83j:57014

23. Goldstone, J.: Unpublished

24. Belavin, A.A., Polyakov, A.M., Schwarz, A.S., Tyupkin, Yu.S.: Pseudoparticle solutions of the Yang-Mills equations. Phys. Lett. 59 B, 85 (1975);

Callan, C.G., Jr., Dashen, R.F., Gross, D.J.: The structure of the gauge theory vacuum. Phys. Lett. 63 B, 334 (1976);

Jackiw, R., Rebbi, C.: Vacuum periodicity in a Yang-Mills quantum theory. Phys. Rev. Lett. 37, 172 (1976);

't Hooft, G.: Symmetry breaking through Bell-Jackiw anomalies. Phys. Rev. Lett. 37, 8 (1976)

25. Freedman, M.H.: J. Differ. Geom. 17, 357 (1982)

26. Milnor, J.: Am. J. Math. 84, 1 (1962)

27. Hitchen, N.: J. Differ. Geom. 9, 435 (1974)

28. Alvarez-Gaumé, L., Della Pietra, S., Moore, G.: Odd dimensional effective actions. Harvard preprint (1984), to appear in Annals of Physics (N.Y.)

29. Atiyah, M.F., Patodi, V.K., Singer, I.M.: Proc. Camb. Philos. Soc. 77, 43 (1975), 78, 405 (1975) 79, 71 (1976) 
30. Alvarez, O., Singer, I.M., Zumino, B.: Gravitational anomalies and the family's index theorem, Commun. Math. Phys. 96, 409 (1984)

31. Witten, E.: Some properties of O(32) superstrings, Phys. Lett. 149 B, 351 (1984)

32. Alvarez-Gaumé, L., Ginsparg, P.: The structure of gauge and gravitational anomalies. Harvard preprint (1984)

33. Ogievetsky, V.I., Polubarinov, I.V.: Sov. J. Nucl. Phys. 4156 (1967); Kalb, M., Ramond, P.: Classical direct interstring action. Phys. Rev. D9, 2273 (1974)

34. Chapline, G.F., Manton, N.S.: Unification of Yang-Mills theory and supergravity in ten dimensions. Phys. Lett. 120 B, 105 (1983)

35. See, for instance, R. Nepomechie, University of Washington preprint (1984)

36. R. Rohm and E. Withen (to appear)

Communicated by A. Jaffe

Received February 5, 1985 
